\title{
Improvement of experimental testing and network training conditions with genome-wide microarrays for more accurate predictions of drug gene targets
}

\author{
Lisa M Christadore', Lisa Pham², Eric D Kolaczyk ${ }^{2,3}$ and Scott E Schaus ${ }^{1,2^{*}}$
}

\begin{abstract}
Background: Genome-wide microarrays have been useful for predicting chemical-genetic interactions at the gene level. However, interpreting genome-wide microarray results can be overwhelming due to the vast output of gene expression data combined with off-target transcriptional responses many times induced by a drug treatment. This study demonstrates how experimental and computational methods can interact with each other, to arrive at more accurate predictions of drug-induced perturbations. We present a two-stage strategy that links microarray experimental testing and network training conditions to predict gene perturbations for a drug with a known mechanism of action in a well-studied organism.
\end{abstract}

Results: S. cerevisiae cells were treated with the antifungal, fluconazole, and expression profiling was conducted under different biological conditions using Affymetrix genome-wide microarrays. Transcripts were filtered with a formal network-based method, sparse simultaneous equation models and Lasso regression (SSEM-Lasso), under different network training conditions. Gene expression results were evaluated using both gene set and single gene target analyses, and the drug's transcriptional effects were narrowed first by pathway and then by individual genes. Variables included: (i) Testing conditions - exposure time and concentration and (ii) Network training conditions - training compendium modifications. Two analyses of SSEM-Lasso output - gene set and single gene - were conducted to gain a better understanding of how SSEM-Lasso predicts perturbation targets.

Conclusions: This study demonstrates that genome-wide microarrays can be optimized using a two-stage strategy for a more in-depth understanding of how a cell manifests biological reactions to a drug treatment at the transcription level. Additionally, a more detailed understanding of how the statistical model, SSEM-Lasso, propagates perturbations through a network of gene regulatory interactions is achieved.

\section{Background}

RNA microarrays have had a major impact on both experimental and computational biology. They have played a role in predicting molecular targets and bioactive compound modes-of-action [1-3], they have helped identify genes responsible for disease- and environmentalinduced phenotypes [4-6]. At the same time, statistical methods for interpreting genome-wide microarray data have progressed over the past decade. Drug target

\footnotetext{
*Correspondence: seschaus@bu.edu

'Department of Chemistry, Boston University, Boston, MA, USA

${ }^{2}$ Program in Bioinformatics and Mathematics, Boston University, Boston, MA, USA

Full list of author information is available at the end of the article
}

identification methods have gone from labor-intensive techniques, like chemogenomic fitness or haploinsufficiency profiling [7-11], to more efficient, statistically driven models such as those based on network-filtering [12-16] and network topology association [17].

Supervised learning methods like support vector machines have also been widely used to develop statistical methods that predict drug-protein interactions [18-22]. These methods employ training networks, constructed from protein-ligand binding data, known protein sequences, compound similarity scores, and in the case of Campillos et al., known drug side effects. Similar to our method, these training networks capture interaction

\section{Biomed Central}


"patterns" between two molecules (eg, ligand-protein) to predict known and new drug targets. Although unlike our method, these patterns are typically taken as known input, whereas in SSEM-Lasso, they are learned from the microarray data.

Accurate interpretation of transcriptional changes resulting from genome-wide microarray data can be influenced by different variables, including those manifested by the experimental biologist and the computational biologist. These variables are especially critical for drug treatment studies, because drugs tend to produce multi-gene and/or off-target perturbations [14,23,24]. For example experimental variables, such as RNA quality, microarray preparation, nutrients, genetic background, and duration and strength of drug treatment can all play a role in the final gene target analysis [25-27]. Similarly, information incorporated into any training, or learning, phase that is used to infer a gene interaction network or similar model structure, can impact results at the gene level. The potential effects of both biological and computational conditions - separately, or on their own - are widely acknowledged. Nevertheless, it appears there is little work explicitly examining how these two types of conditions interact with each other to produce accurate and reliable molecular target predictions.

SSEM-Lasso uses a network-based approach consisting of two phases, training and testing. In the training phase, the method learns a collection of gene-gene interaction effects from compendium of microarray experiments (training compendium), which are captured in an interaction network (Figure 1A). Then, in the testing phase, the method identifies genes experiencing an additive shift in their mean transcript levels in response to an external perturbation (eg, drug treatment), after adjusting for the inferred gene-gene interactions (Figure 1B). Transcript residuals resulting from this step are ranked by their absolute values for all annotated genes in the compendium (Figure 1B). Genes with low ranks (large residuals) are genes SSEM-Lasso distinguishes as standing out from the background gene regulatory effects. They are flagged as potential targets of the external perturbation of interest. As a result, SSEM-Lasso has the ability to significantly narrow the gene target window in comparison to RNA change z-score computations (Figure 1C).

SSEM-Lasso is unlike standard machine learning methods in that it is based on an explicit model of how perturbations propagate through a network of gene regulatory effects, in the form of a system of sparse simultaneous equations (which, in turn, may be viewed as a stochastic version of standard first-order differential equations) [12]. Other machine learning approaches typically have been based on models defined through relationships learned between known drug-target interactions and various biological inputs [18-22]. SSEMLasso also differs from machine learning in the data being analyzed and the interaction pairs being identified. SSEM-Lasso uses microarray gene expression data from hundreds of experiments carried out under varying experimental conditions. The machine learning methods discussed above use for example, protein-ligand interaction and compound 2-D similarity data listed in conventional databases like KEGG [28], BRENDA [29], and SIMCOMP [30].

SSEM-Lasso's mathematical properties have been fully characterized [12]. The method has achieved improved sensitivity and accuracy over the RNA z-test method (ie, ranking genes as targets based on normalized expression data, without any additional modelling or processing) and a competitive, alternative network-inference method, MNI [14]. Furthermore, the two phases of SSEM-Lasso allow for modifications of both experimental variables (testing phase) and computational variables (training phase) to optimize drug target predictions.

SSEM-Lasso is applicable to a range of perturbation experiments. The method does not impose explicit constraints on the experimental conditions, such as particular deletion strain, inducible expression system, or time-course conditions, for inferring gene network interactions. SSEM-Lasso has been shown to perform well in the context of in silico experiments. Furthermore, it has proven successful at predicting gene targets of $S$. cerevisiae haploid and diploid deletion strains. However, the method requires further optimization for predicting drug gene targets (Additional file 1). This is an anticipated caveat due to the biologically complex nature of a chemical perturbation. Given this combination of features, we determine SSEM-Lasso ideal for our goal of investigating experimental-computational dynamics, and at the same time, we seek to improve its performance with drug gene target predictions.

We use the model organism, S. cerevisiae, and a compound with a known mechanism of action, fluconazole (FL), to explore how choice of variables in testing and training phases influences the quality of final gene target predictions. FL specifically binds and inhibits cytochrome P450 (CYP450)-dependent lanosterol C-14- $\alpha$ demethylase (Erg11p) [31,32], an essential hemoprotein in the ergosterol biosynthesis pathway (Figure 2). Ergosterol is the principle component of yeast cell membranes, similar to cholesterol in animal cells. FL inhibition of Erg11p causes the accumulation of toxic $14-\alpha-$ methylated sterols and ergosterol depletion [33,34]. This results in increased cell membrane permeability and asymmetry and irregular sphingolipid, phospholipid and long-chain fatty acid synthesis [35-37]. Additionally, Erg11p is dependent on oxygen and heme production, 


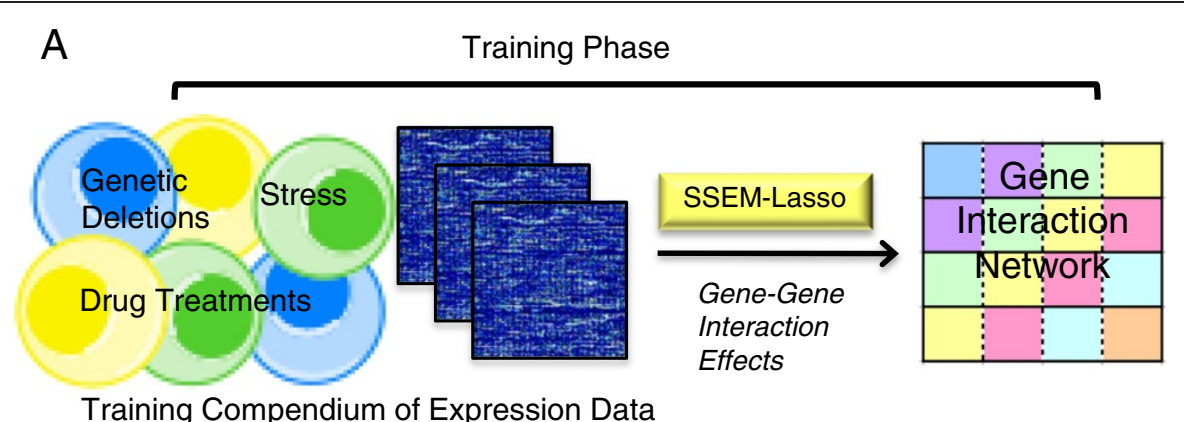

B

Testing Phase

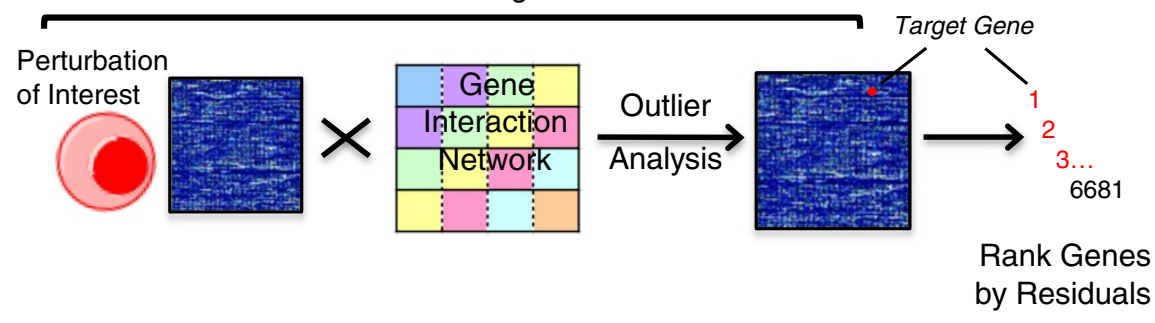

C
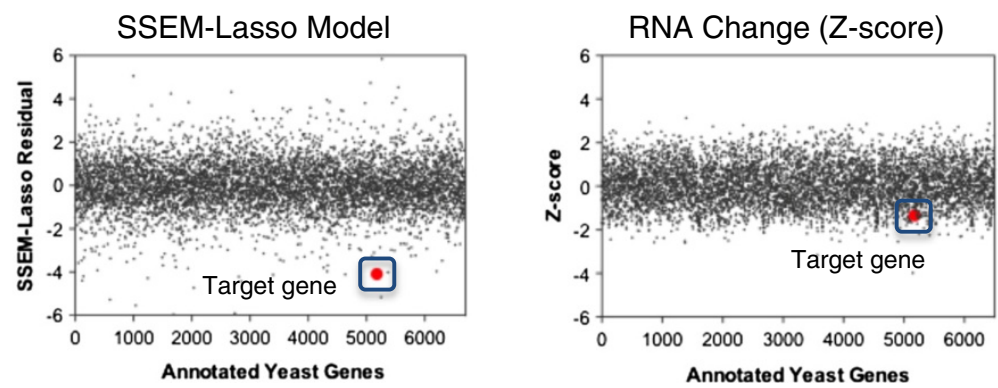

Figure 1 SSEM-Lasso network-inference methodology for prediction of gene targets. (A) In the training phase, transcript signals derived from a training compendium of Affymetrix yeast expression data estimated a gene interaction network using sparse simultaneous equation models and Lasso regression (SSEM-Lasso). The gene interaction network accounted for every gene's effect on another gene within the compendium and was used to infer subsequent experimental perturbations of interest. (B) In the testing phase, experimental expression data was processed with the gene interaction network, and mRNA transcript signals were adjusted based on all inferred gene regulatory effects in the network. An outlier analysis yielded residual values for every gene in the compendium. Residuals were ranked by their absolute values, and genes with lower ranks were considered more accurate predictions of directly targeted genes of the experimental perturbation. (C) SSEM-Lasso "resolves" experimentally perturbed genes out of the background gene-gene interaction "noise" in the network. This results in a more stringent gene-target filter in comparison to standard z-score computation. The data shown is from a top2 $2 / T O P 2$ heterozygous yeast deletion microarray experiment conducted inhouse. The gene target, TOP2, is significantly perturbed when evaluated with SSEM-Lasso compared to the RNA z-score prediction.

and ERG11 deletion strains are nonviable under aerobic growth conditions. Therefore, Erg11p disruption by FL can also lead to defects in heme biosynthesis and mitochondrial respiration [38-40].

In addition to ERG11, ERG6, UPC2 and HAP1 are impacted by sterol and heme depletion. ERG6 encodes for $\Delta(24)$-sterol C-methyltransferase, an enzyme that functions downstream of Erg11p in ergosterol biosynthesis and is responsible for the transmethylation of zymosterol to fecosterol [36] (Figure 2). Although Erg6p is not the direct gene target of FL, nor is it essential for cell growth, Erg6p regulates appropriate sterol and fatty acid composition and distribution, and is therefore required for normal cell membrane permeability and rigidity [41-43].

Sterol regulatory element binding protein, Upc2p, is a member of the $\mathrm{Zn}_{2}-\mathrm{Cys}_{6}$ transcription factor family required for exogenous sterol uptake during anaerobic growth. Upc2p, with Ecm22p, regulates the transcription of late stage ergosterol biosynthesis genes in response to azole-induced sterol depletion [44,45] (Figure 2). Its DNA binding sequence is conserved between $C$. albicans 


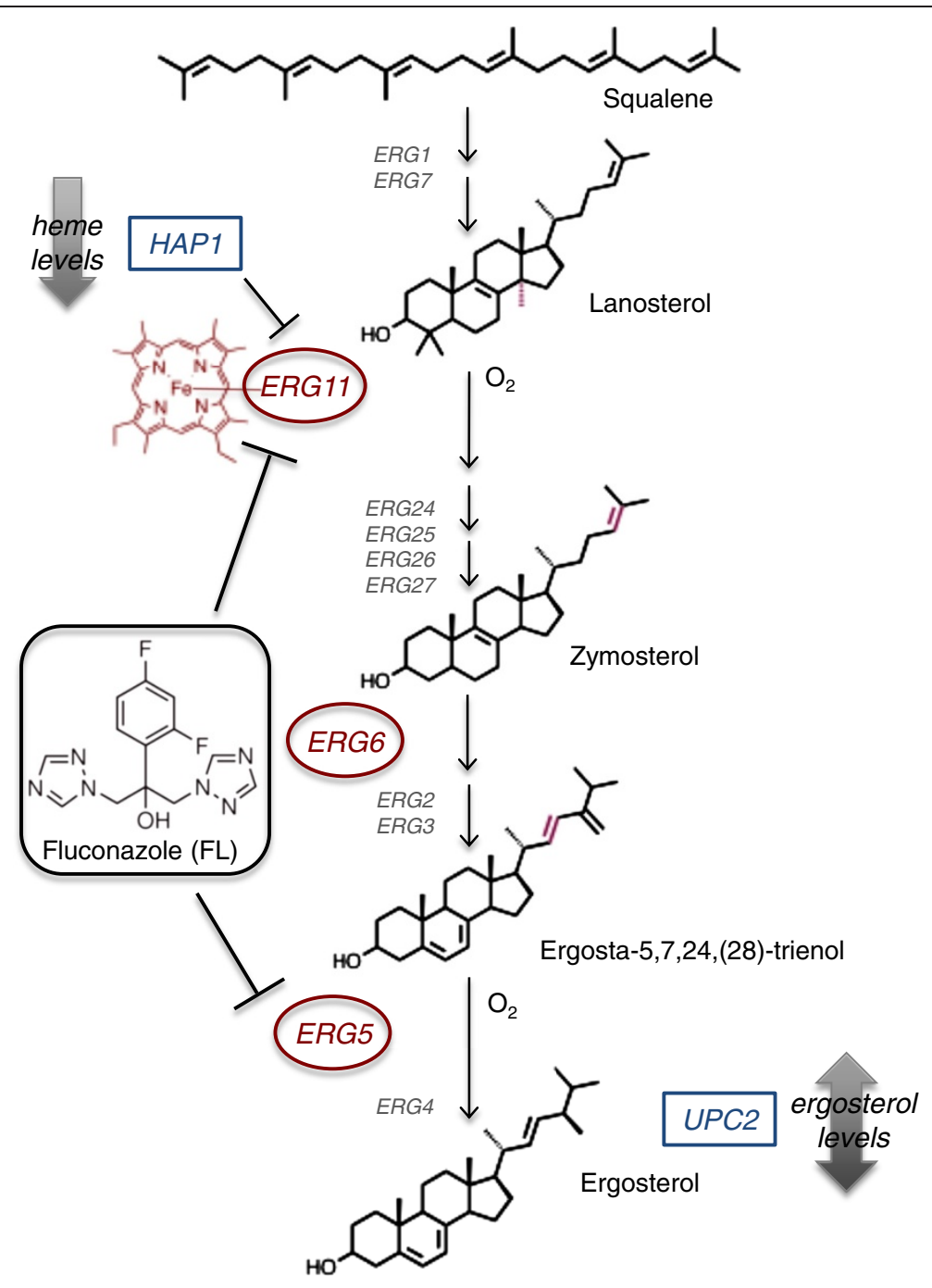

Figure 2 Summary of FL enzymatic and transcription factor gene targets. Genes affected by fluconazole (FL) investigated in this study are enzymes along the ergosterol biosynthetic pathway (circles) and transcription factors directly regulated by sterol and heme levels (squares). ERG 11, the gene that codes for lanosterol C-14-a demethylase, is the primary target of FL. CYP450 C-22 sterol desaturase, ERG5 (circle), is also a target of FL and its enzymatic activity is inhibited upon FL binding. FL's nitrogen interacts with the heme groups of both Erg11p and Erg5p disrupting normal ergosterol synthesis and affecting downstream enzymatic reactions, including those performed by $\Delta[24]$-sterol C-methyltransferase, Erg6p (circle). FL disruption of sterol biosynthesis additionally affects UPC2 (square), the gene that encodes for a sterol regulatory binding protein responsible for increased transcription of ERG genes upon sterol depletion. FL induces defective respiration due to its disruption of heme and oxygen levels. Therefore, HAP1 (square), a transcription factor responsible for regulating ERG11 expression under hypoxic conditions, is also targeted.

and S. cerevisiae and is in a region of the ERG11 promoter critical for azole induction of ERG11 expression $[46,47]$.

Heme-activator protein, Hap1p, is a transcription factor that controls the expression of aerobic and anaerobic genes through both its interactions with heme and transcriptional control of heme-dependent repressor of hypoxic genes, [48,49]. Hap1p binds the promoters of ERG5 and ERG11 under both aerobic and hypoxic conditions, however it is most active in repressing these genes' expressions under hypoxic conditions [48,50] (Figure 2). ERG11 expression through induction of ROX1 is also regulated by Hap1p. Additionally, Hap1p controls Upc2p expression to maintain basal expression levels of $E R G$ genes. However upon sterol depletion, Upc2p no longer requires Haplp to transcribe ERG genes [51]. These genes - ERG6, UPC2, and HAP1 - are therefore interconnected and exemplary targets to further investigate FL-induced expression changes.

The expression of the CYP450 C-22 sterol desaturase, $E R G 5$, is indirectly investigated in this study through the haploid heme deletion strain, hem1 $1 \Delta$. The hem1 $\Delta$ strain cannot synthesize $\delta$-aminolevulinic acid, a precursor in heme synthesis. In the absence of heme, Hap1p binds 
the ERG5 promoter to suppress expression of ERG5. Therefore hem1 $\Delta$ mutants contain little to no Erg5p mRNAs [48]. Additionally, Erg5p can be directly bound and inhibited by azoles, like FL (Figure 2) [52]. Thus, ERG5 is considered the primary target of the hem1A strain and an additional FL-targeted gene in this study.

This study contributes to the larger goal of improving microarrays for therapeutic discovery and development. Using a model organism and an established drug with known target genes and pathways, we are able to identify conditions that both the experimentalist and computational biologists can fine-tune to more accurately predict drug effects at the gene expression level. Herein we present a step-wise strategy through SSEM-Lasso that scientists can take to optimize drug gene target predictions at the genome-wide level.

\section{Results}

Testing phase (experimental) variations for predicting FL gene targets

The overall goal of the testing phase was to determine if changes in experimental variables, or "input", altered SSEM-Lasso ranks, or "output", of drug gene targets (Figure 1B). We assessed how well SSEM-Lasso identified a target gene relative to off -target, or orthogonal, genes over changing experimental conditions. This was evaluated by comparing the rank of the target gene relative to the rank of off-target genes.

Studies have shown that desired physiological responses can be achieved by controlling the duration a drug is in contact with the host organism and the concentration of drug administered. This in turn can lead to more effective treatment strategies [53,54]. In the testing phase we regulated these critical treatment variables, exposure time and concentration, while maintaining constant the training compendium used to infer the gene network interactions. Figure 3 outlines the experimental workflow. Wild-type $S$. cerevisiae cells were treated with FL and harvested at either varying exposure times (ET) or concentrations in aerobic, batch culture conditions (Figure 3A). Affymetrix microarray experiments were carried out in duplicate, and transcript signals were RMA-normalized and processed with SSEM-Lasso (Figure 3B). For all testing phase variations, the original training compendium from Cosgrove et al. was used to infer the gene interaction network.

Two analyses of SSEM-Lasso output - gene set and single gene - were conducted. For the gene set analysis, genes representative of 6 different biological pathways were grouped to form 1 target and 5 orthogonal gene sets. The target gene set unique to FL action, called "FL-interacters", was comprised of genes affected by FL and/or associated with ERG11. FL-interacters included genes from ergosterol, terpenoid backbone, fatty acid and sphingolipid biosynthesis pathways, and sterol transport, heme and oxygen sensing pathways (Additional file 2). The five orthogonal gene sets included genes from: DNA replication and repair, pyrimidine biosynthesis and metabolism, RNA transport, glycolysis and pentose phosphate and mitosis (Additional files 3, 4, 5, 6, 7).

Gene set analysis was carried out as follows: Each gene within a gene set was detected across an increasing gene rank threshold, resulting in a receiver operating characteristic, or ROC, curve for an FL treatment experiment. ROC curves are graphical plots of the true positive rate against the false positive rate as the discrimination threshold is varied. Areas under ROC curves were converted into percentages (AUC\%) and compared across FL treatments (Figure 3C). Steeper ROC curves result in larger AUC percentages. The expected AUC from random guessing is 0.5 , or $50 \%$. AUC values falling below 0.5 denoted a higher false positive rate than random guessing, while AUC values above 0.5 denoted more true positives than what were expected with random guessing. We considered treatment experiments with AUCs $>0.5$ to experience stronger biological pathway effects than experiments with AUC\%s $\leq 0.5$.

For single gene analysis, FL perturbation of its primary target, ERG11, was tracked along with FL targets associated with ergosterol biosynthesis, sterol transcription initiation and heme and oxygen transcriptional regulation, ERG6, UPC2 and HAP1, respectively (Figure 3D). Ranks for orthogonal genes essential to cellular survival, MPS1, $A D E 13, T O P 2, C D C 9, P A B 1$ and $U B A 1$, were also monitored as indicators of FL off-target effects. Lower ranks suggested more accurate prediction of FL action on an individual gene (Figure 3D). Rank percentiles were also computed to assess how well a gene ranked relative to the background set of all genes.

\section{Training phase (network inference) variations for improving $\mathrm{FL}$ single gene target predictions}

In addition to the testing phase strategy, modifications to the gene interaction network in the training phase were conducted. The overall goal of the training phase was to determine if changes to the network increased or decreased ranks of gene targets.

Variations to the training phase involved the addition of biologically-motivated microarray expression data into the original training compendium from Cosgrove et al. Subsequent modifications to gene-gene interaction "patterns" propagated through the network resulted in changes of varying degrees in SSEM-Lasso rank predictions (Figure 4). Two measurements, rank change (RC) and RC percentile, reflected how strongly network variations affected SSEMLasso predictions. If $\mathrm{RC}$ of a target gene was positive, prediction of the gene perturbation was said to "improve". RC of a target gene was compared against all gene RCs within an experiment by calculating a percentile of $\mathrm{RC}$, or the 


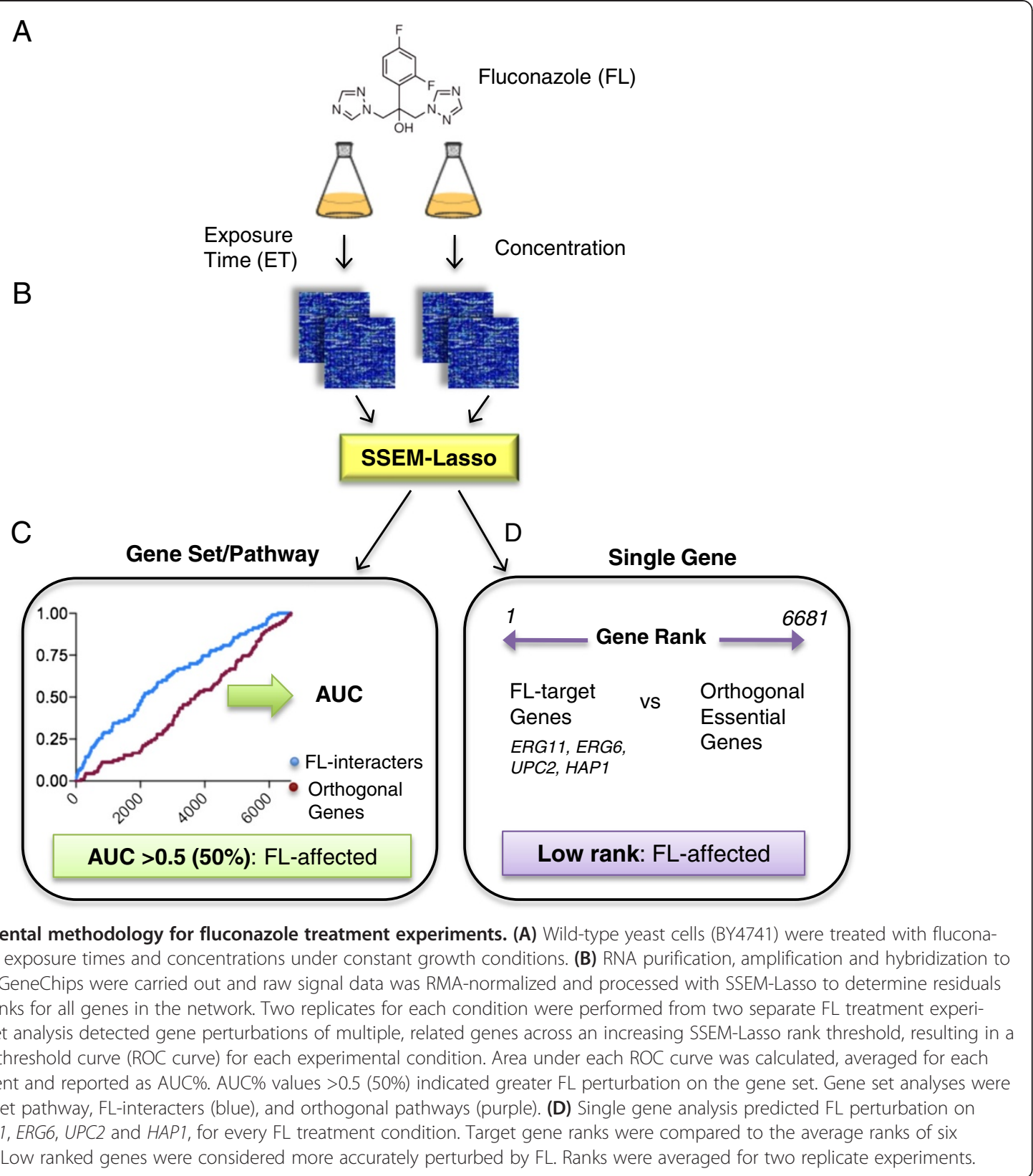

percentage of genes with a $\mathrm{RC}$ as high or higher than the target gene. The $\mathrm{RC}$ percentile provide a quantitative assessment of RC. Thus, a tuneable network inference variable was established in the training phase.

\section{Testing phase: Exposure time variations have stronger} effects on FL gene set than on FL single gene targets The first experimental condition manipulated in the testing phase was FL exposure time (ET). The length of FL treatment in a population of unsynchronized cells was varied between one and four ETs, at constant FL concentration $\left(\mathrm{GI}_{10}\right)$. For each ET experiment, results from both gene set
(AUC\%) and single gene (rank and percentile) were reported.

Gene set analysis for FL-interacting genes resulted in individual AUC\%s for each ET experiment, which were plotted for each of the 6 gene sets as shown in Figure 5. Longer FL ETs correlated with higher AUC\%s. From one to two ETs, AUC\% increased noticeably from 50 to $55 \%$, and at four ETs AUC\% was at its highest of 60\% (Figure 5, squares). These results indicated that incubation of cells with FL for four ETs was the optimal time point for SSEMLasso to predict FL effects on multiple, target genes.

AUC\%s for the orthogonal gene sets were also plotted, and similar improvement trends were observed for mitosis 


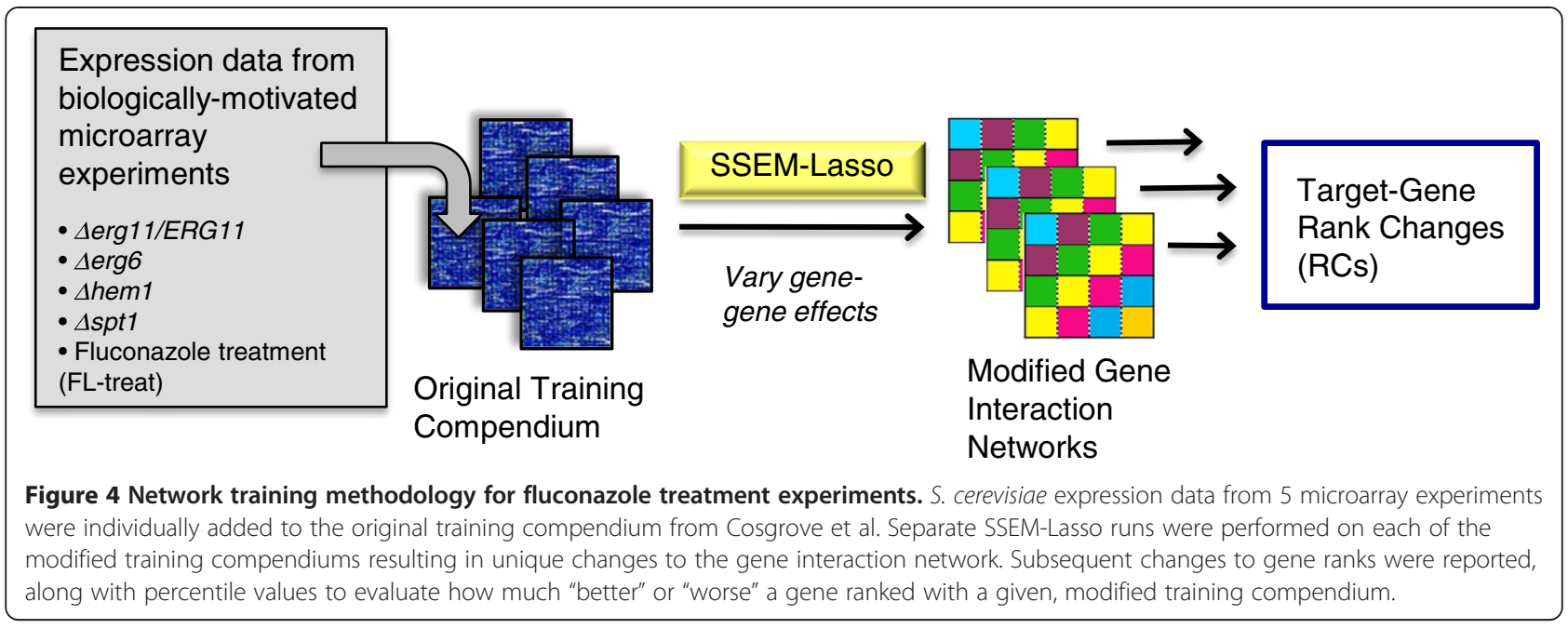

and glycolysis/pentose phosphate gene sets (Figure 5, diamonds and inverted triangles). SSEM-Lasso likely identified similar trends for these pathways, because they are also influenced by FL treatment. Severe ergosterol depletion interferes with sparking functions of ergosterol [55]. Hence, genes involved in mitosis that are critical to late stages of the cell cycle may have been dysregulated upon FL treatment. Additionally, FL interrupts heme function, and thus the cell population may have adapted to increased hypoxic conditions by shifting from glucose metabolism via respiration to anaerobic fermentation [56], thus affecting genes involved in glycolysis/pentose phosphate. Yet, the other orthogonal pathways, RNA transport, pyrimidine biosynthesis/metabolism, and DNA replication/repair, did not reproduce the same improvement trend nor exhibit AUC\% values $>50 \%$ (Figure 5). Overall, the average AUC\%s for each of the 5 orthogonal gene sets were lower than those for FL-interacter gene set, demonstrating that FL exerted its strongest effects on the FL-targeted gene set (Figure 5).
Single gene targets also were tracked across ETs. ERG11 ranked consistently lower than the collective population of orthogonal genes (Figure 6A), suggesting SSEM-Lasso's predictions were more specific for FL action on its therapeutic target.

Interestingly, changing ET $(1 \rightarrow 4)$ did not improve the algorithm's predictions, and ERG11 rank remained constant, between 1716 and 2080 (Figure 6A, squares), or in the $70^{\text {th }}$ percentile of ranks (Table 1). This was unlike the FL-interacter gene set results, which demonstrated a distinct trend with increasing ET (Figure 5). ERG6 was the only FL target that followed a distinct trend, in which ERG6 rank decreased at higher ET. At three and four ETs, ERG6 ranked in the 78th and 87th percentiles respectively, which were lower than ERG11 ranks (Figure 6B, triangles, Table 1). Ranks for UPC2 and HAP1 were consistently lower than both ERG11 and ERG6. UPC2 and HAP1 ranked in the $93^{\text {rd }}$ to $99.7^{\text {th }}$ percentiles for two, three, and four ETs. Similar to ERG11,

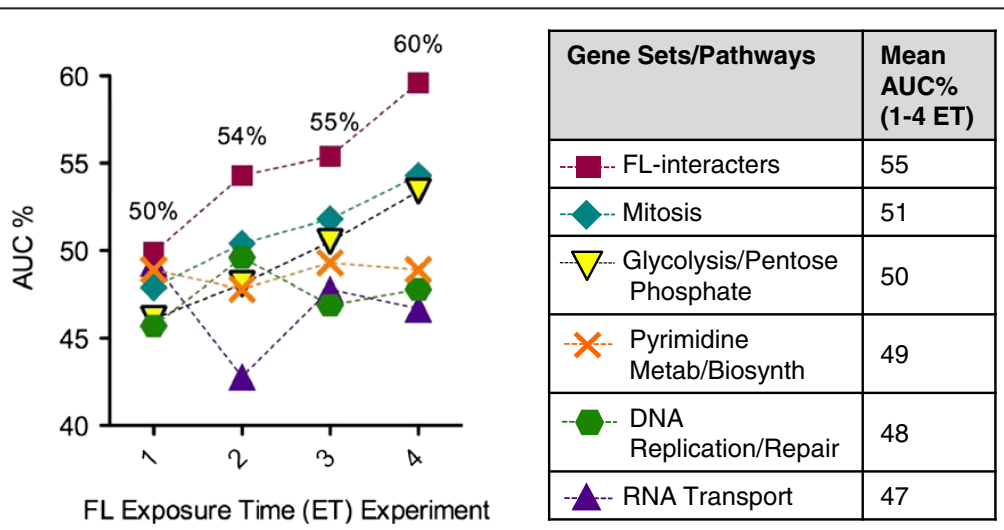

Figure 5 Exposure time effects on gene set (AUC\%) analysis. Areas under each sensitivity vs. rank threshold curve (ROC curve) for FLinteracters and orthogonal gene sets/pathways were converted to percentages (AUC\%s) and plotted for each FL ET experiment. Mean AUC\%s (ET 1 to 4) for each gene set were computed and compared in the table. Larger AUC\% values indicated better prediction of FL action on a gene set. AUC\% values were the averages of two replicates. 

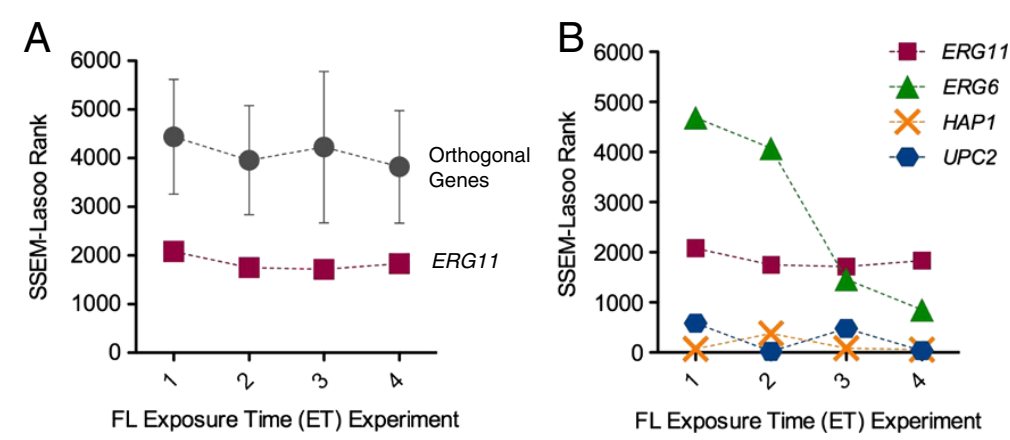

Figure 6 Exposure time effects on single gene (rank) analysis. (A) SSEM-Lasso ranks of FL's primary gene target, ERG11 (squares), were compared to gene rank averages for six orthogonal genes, MPS1, ADE13, TOP2, CDC9, PAB1 and UBA1 (circles), across increasing ETs. Error bars represent standard deviation for orthogonal gene ranks. (B) SSEM-Lasso ranks of all FL targets, ERG11 (squares), ERG6 (triangles), UPC2 (hexagons) and HAP1 (crosses) versus FL ET experiments. Cells were treated with FL concentrations that corresponded to increasing growth inhibitory percentages, GI\%s (x-axis). Lower ranks indicated better prediction of $\mathrm{FL}$ action on an individual gene. All ranks were the averages of two replicates.

UPC2 and HAP1 ranks did not vary with changes to ET. (Figure 6B, hexagons and crosses and Table 1).

\section{Testing phase: Concentration changes have minimal to modest effects on FL gene target predictions}

The second testing phase variable optimized was FL concentration. S. cerevisiae cells require ergosterol for normal aerobic growth [39], so it was necessary to determine a treatment concentration at which wild-type cells continued dividing with impaired ergosterol synthesis. FL doseresponse curves and growth inhibitory values (GIs) were generated from in-house cell growth inhibition assays. FL dosing strategy was based on concentrations below the $\mathrm{GI}_{50}$ value $-\mathrm{GI}_{0.5}, \mathrm{GI}_{5}, \mathrm{GI}_{10}, \mathrm{GI}_{20}, \mathrm{GI}_{30}$ and $\mathrm{GI}_{40}$ - so that FL would not inhibit growth of more than $50 \%$ the cell population. Thus, gene perturbations were not considered direct results of cell death signalling pathways, but steadystate expression changes specific to FL. SSEM-Lasso results were evaluated within the same gene set and single gene analysis frameworks as the ET experiments.

Similar to the FL gene set trend for ET, higher FL treatment concentrations improved AUC\% values. FL effects were obvious upon treatment, jumping from $51 \%$ for control to $64 \%$ for $\mathrm{GI}_{0.5}$ (Figure 7). AUC\%s continued to improve as $\mathrm{FL}$ concentrations increased, up to $\mathrm{GI}_{20}$. These results demonstrated that SSEM-Lasso predicted FL action more accurately at treatment concentrations between $\mathrm{GI}_{20}$ and $\mathrm{GI}_{40}$, with $\mathrm{AUC} \%$ s reaching 67-69\% (Figure 7, squares). Orthogonal gene sets failed to show a similar trend as the FL-interacter gene set nor did they experience enhanced perturbation upon FL treatment. Average AUC\% values remained below the FL-interacter gene set average of $64 \%$ across all FL concentrations (Figure 7). This was a positive indication that FL was specifically targeting genes associated with its activity.

In general, FL single gene ranks were insensitive to changes in FL concentrations, just as they did not fluctuate significantly with changing ETs. At the onset of FL treatment, ERG11 rank dropped from 5714 to 1812, a clear response to FL treatment. However over increasing treatment concentrations, ERG11 ranks hovered modestly between $1800-2300$ (ranks in the $\sim 70^{\text {th }}$ percentile) with no noteworthy fluctuations (Figure $8 \mathrm{~A}$, diamonds and Table 2). Average ranks of the six orthogonal genes were predicted with SSEM-Lasso and proved unperturbed by FL. In addition, the orthogonal genes failed to experience the pronounced rank increase ERG11 had at the onset of FL treatment (Figure 8A, circles), suggesting SSEM-Lasso was accurately predicting FL effects on its target.

$E R G 6$ followed a very similar concentration trend but with overall lower ranks than ERG11 (Figure 8B, triangles). Transcription factors, UPC2 and HAP1, had

Table 1 Comparison of single gene ranks for FL targets across increasing ETs

\begin{tabular}{|c|c|c|c|c|c|c|c|c|}
\hline \multirow[b]{2}{*}{ ET Expt } & \multicolumn{2}{|c|}{ ERG11 } & \multicolumn{2}{|c|}{ ERG6 } & \multicolumn{2}{|c|}{ UPC2 } & \multicolumn{2}{|c|}{ HAP1 } \\
\hline & Rank & Percentile & Rank & Percentile & Rank & Percentile & Rank & Percentile \\
\hline $1 \mathrm{ET}$ & 2080 & 69 & 4686 & 30 & 588 & 91 & 859 & 87 \\
\hline $2 \mathrm{ET}$ & 1752 & 74 & 4078 & 39 & 20 & 99.7 & 426 & 94 \\
\hline $3 \mathrm{ET}$ & 1716 & 74 & 1461 & 78 & 483 & 93 & 291 & 96 \\
\hline $4 \mathrm{ET}$ & 1835 & 73 & 852 & 87 & 39 & 99 & 56 & 99 \\
\hline
\end{tabular}

Ranks for each FL gene target were reported for each ET. Ranks are the average of two replicate experiments. Rank percentile was computed as the percentage of genes with a rank as high or higher than the target gene. Rank percentiles $\geq 95$ indicated significant perturbation of a gene as identified by SSEM-Lasso. 


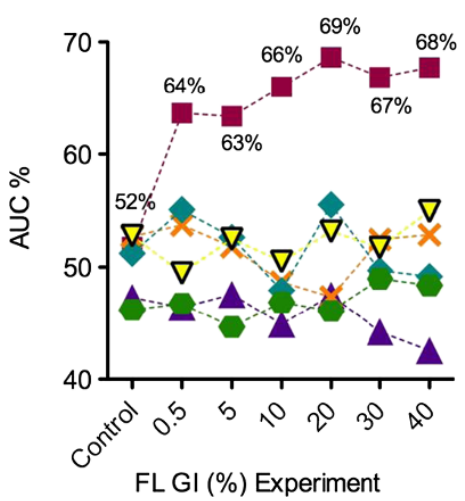

\begin{tabular}{|c|c|}
\hline Gene Sets/Pathways & $\begin{array}{l}\text { Mean } \\
\text { AUC\% } \\
\text { (1-4 ET) }\end{array}$ \\
\hline -1- FL-interacters & 64 \\
\hline Mitosis & 52 \\
\hline $\begin{array}{l}\nabla \text { Glycolysis/Pentose } \\
\text { Phosphate }\end{array}$ & 52 \\
\hline $\begin{array}{l}\text { - Pyrimidine } \\
\text { Metab/Biosynth }\end{array}$ & 51 \\
\hline $\begin{array}{l}\text { DNA } \\
\text { Replication/Repair }\end{array}$ & 47 \\
\hline - RNA Transport & 46 \\
\hline
\end{tabular}

Figure 7 Concentration effects on gene set (AUC\%) analysis. Areas under each sensitivity vs. rank threshold curve (ROC curve) for FLinteracters and orthogonal gene sets were converted to percentages (AUC\%s) and plotted for each FL microarray concentration experiment. Cells were treated with FL concentrations that corresponded to increasing growth inhibitory percentages, $\mathrm{Gl} \% \mathrm{~s}$ (x-axis). Mean $\mathrm{AUCC}_{\mathrm{s}}\left(\mathrm{Gl} \mathrm{l}_{0.5}\right.$ to $\mathrm{Gl}_{40}$ ) for each gene set were computed and compared in the table. Larger AUC\% values indicated better prediction of FL action on a gene set. AUC\% values were the averages of two replicates.

overall ranks below 200 , or in $98^{\text {th }}$ to $99^{\text {th }}$ percentile (with the exception of UPC2 for $\mathrm{GI}_{0.5}$ experiment). However, a pronounced trend over increasing concentrations was not observed (Figure 8B, hexagons and crosses and Table 2). These results were consistent with the ET results, wherein the FL-targeted transcription factors performed significantly better than the metabolic targets, $E R G 11$ and $E R G 6$, and also were not as strongly affected by ET and concentration changes as ERG11 and ERG6.

Training phase: Input of microarray expression data and modification of the gene interaction network influences FL single gene target predictions

After exploring how experimental testing conditions influenced predictions of drug target predictions, focus shifted to the computational details of SSEM-Lasso's training phase. Specifically, we generated modified training compendiums, or unique training phase variables, and examined how they altered the network's gene-gene interaction "patterns" to improve final gene ranks. Previous SSEMLasso studies demonstrated that removal of experiments in the same project group from the training compendium boosted SSEM-Lasso performance, perhaps due to the reduction of artifactual effects caused by lab-specific experimental variables [12]. Here, we were interested in how the addition of microarray experiments related to the applied perturbation, FL treatment, affected predictions of $\mathrm{FL}$ gene targets.

Taking advantage of the Saccharomyces genome deletion collection $[57,58]$, microarray expression data was obtained for three deletion strains related to FL mechanism of action, $\operatorname{erg} 11 \Delta / E R G 11$, erg6 6 , hem1 15 [5], and
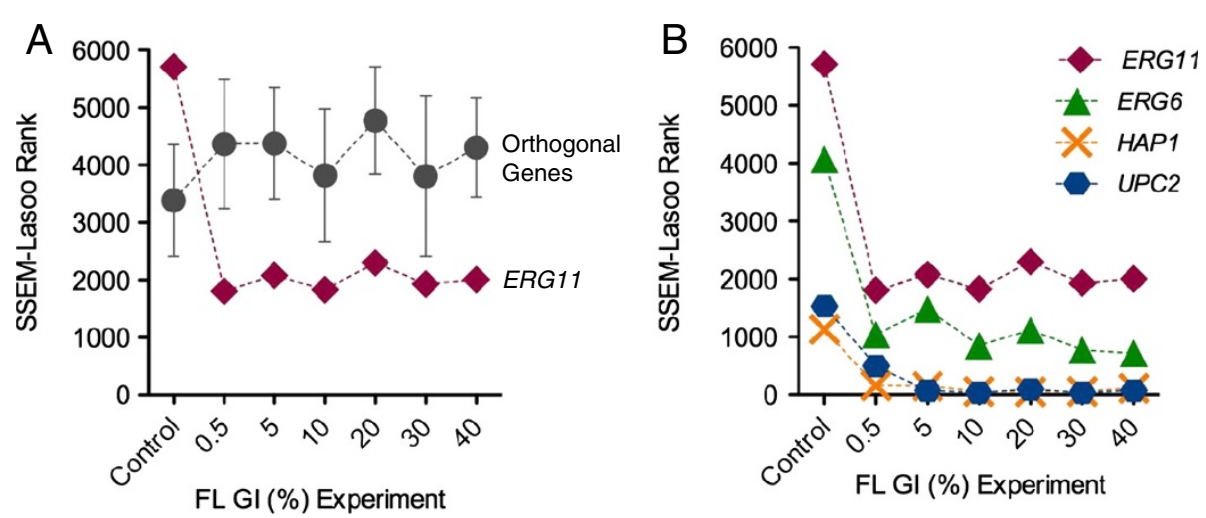

Figure 8 Concentration effects on single gene (rank) analysis. (A) SSEM-Lasso ranks of FL's primary gene target, ERG11 (diamonds), were compared to gene rank averages for six orthogonal genes, MPS1, ADE13, TOP2, CDC9, PAB1 and UBA1 (circles), across increasing FL concentrations. Error bars represent standard deviation for orthogonal genes. (B) SSEM-Lasso ranks of all FL targets, ERG11 (diamonds), ERG6 (triangles), UPC2 (hexagons) and HAP1 (crosses) versus concentration experiments. Cells were treated with FL concentrations that corresponded to increasing growth inhibitory percentages, Gl\%s (x-axis). Lower ranks indicated better prediction of FL action on an individual gene. All ranks are the averages of two replicates. 
Table 2 Comparison of single gene ranks for FL targets across increasing concencrations (GI\%s)

\begin{tabular}{|c|c|c|c|c|c|c|c|c|}
\hline \multirow[b]{2}{*}{ GI Expt } & \multicolumn{2}{|c|}{ ERG11 } & \multicolumn{2}{|c|}{ ERG6 } & \multicolumn{2}{|c|}{ UPC2 } & \multicolumn{2}{|c|}{ HAP1 } \\
\hline & Rank & Percentile & Rank & Percentile & Rank & Percentile & Rank & Percentile \\
\hline Control & 5714 & 14 & 4068 & 39 & 1543 & 77 & 1133 & 83 \\
\hline $\mathrm{Gl}_{0.5}$ & 1812 & 73 & 1047 & 84 & 498 & 93 & 160 & 98 \\
\hline $\mathrm{Gl}_{5}$ & 2081 & 69 & 1489 & 78 & 73 & 99 & 149 & 98 \\
\hline $\mathrm{Gl}_{10}$ & 1835 & 73 & 852 & 87 & 39 & 99 & 56 & 99 \\
\hline $\mathrm{Gl}_{20}$ & 2302 & 66 & 1119 & 83 & 93 & 99 & 105 & 98 \\
\hline $\mathrm{Gl}_{30}$ & 1934 & 71 & 775 & 88 & 40 & 99 & 66 & 99 \\
\hline $\mathrm{Gl}_{40}$ & 2007 & 70 & 721 & 89 & 74 & 99 & 116 & 98 \\
\hline
\end{tabular}

Ranks for each FL gene target were reported for each concentration. Ranks are the average of two replicate experiments. Rank percentile was computed as the percentage of genes with a rank as high or higher than the target gene. Rank percentiles $\geq 95$ indicated significant perturbation of a gene as identified by SSEM-Lasso.

one not linked to FL action, spt3ム [60]. Expression data from the FL drug treatment that yielded the best predictions of ERG11 perturbation $\left(\mathrm{GI}_{10} 4 \mathrm{ET}\right)$ was also included in training phase variations. First, we confirmed SSEM-Lasso identified the direct gene target for each deletion strain. In agreement with previous studies, SSEM-Lasso predicted all genetic deletion targets accurately (Table 3 ). We proceeded to individually add each experiment's expression data into the original training compendium to generate five new training compendiums. Modified gene interaction networks were then inferred for each modified training compendium (Figure 4). Changes in gene rank (RC) between the original and modified training compendiums for $\mathrm{FL}$ targets, ERG11, ERG6, ERG5, and non-target SPT3, were subsequently determined.

Graphs in Figure 9A-D display five representative FL treatment experiments on the $\mathrm{x}$-axis and $\mathrm{RC}$ values for ERG11, ERG6, ERG5 or SPT3 on the y-axis. These results showed that each modification to the training compendium induced changes to the gene network that were unique to the deletion strain's corresponding target gene. ERG11, ERG6, and ERG5 ranks dropped, or improved, only with the addition of expression data from erg11 / ERG11, erg6 6 , and hem1A, respectively. In contrast to the addition of genetic deletions, inclusion of expression data from the FL treatment experiment did not impact network interactions to alter ERG11, ERG6 and ERG5 ranks. In most cases, ranks stayed the same or worsened with the addition of FL drug treatment expression data (Figure 9A-C, Tables 4, 5 and 6).

ERG11 RCs for all FL treatment experiments dropped substantially when ERG11 deletion expression data was added to the training compendium. ERG11 ranks were in the $97^{\text {th }}, 92^{\text {th }}, 96^{\text {th }}, 88^{\text {th }}$, or $94^{\text {th }}$ percentile of RCs for each of the five experiments (Table 4). Inclusion of ERG6 deletion expression data into the training compendium also improved prediction of ERG6 perturbations across all FL experiments. In fact, inclusion of the haploid erg6 $\Delta$ experiment to the training compendium produced a more pronounced drop in ERG6 ranks than the heterozygous erg11 $1 / E R G 11$ experiment did for ERG11 ranks. ERG6 RC percentiles were in the range of 97 to 100 , with the exception of the $\mathrm{FL}_{\mathrm{GI} 40}$, 4DT experiment (Table 5). Addition of hem1 $\Delta$ expression data also improved ERG5 ranks for all FL treatment experiments, and ERG5 RCs were in the $97^{\text {th }}, 75^{\text {th }}, 97^{\text {th }}, 95^{\text {th }}$, or $90^{\text {th }}$ percentiles (Table 6). ERG5 rank improvements were similar in magnitude and direction to those of ERG11. This result suggested that ERG11 and ERG5 were similarly affected by FL, and also by heme depletion, supporting their synergy along the ergosterol biosynthesis pathway and FL's interactions with their CYP heme centers.

To test if network changes induced by erg11 $1 / E R G 11$, erg6 $\Delta$ and hem1 $\Delta$ were specific to $\mathrm{FL}$, we analyzed whether haploid spt3 $\Delta$ expression data improved SPT3 ranks for the same FL experiments. Spt3p, a subunit of SAGA-type histone acetyltransferase complex, is not specifically targeted by FL. Indeed, inclusion of spt3A

Table 3 Gene ranks predicted by SSEM-Lasso for corresponding yeast genetic deletion microarray experiments

\begin{tabular}{llll}
\hline $\begin{array}{l}\text { Microarray } \\
\text { experiment }\end{array}$ & $\begin{array}{l}\text { Gene } \\
\text { target }\end{array}$ & $\begin{array}{l}\text { Rank with original } \\
\text { compendium }\end{array}$ & $\begin{array}{l}\text { Rank with modified } \\
\text { compendium }\end{array}$ \\
\hline erg11 1 ERG $11^{1}$ & ERG11 & 277 & 301 \\
erg6 ${ }^{1}$ & ERG6 & 1 & 1 \\
spt3 $\Delta^{2}$ & SPT3 & 5 & 47 \\
hem $1 \Delta^{3}$ & ERG5 & 40 & 41 \\
\hline
\end{tabular}

${ }^{1}$ Christadore, L. Boston University. 2012.

2James, N., et al. Genetics 177:123 2007 [60].

${ }^{3}$ Protchenko O., et al. Eukaryot. Cell 7:859 2008 [59].

SSEM-Lasso gene ranks were determined for gene targets of genetic deletions and compound treatments. All microarray experiments were performed using Affymetrix Yeast Genome 98 gene chips, and data was RMA-normalized before processing with SSEM-Lasso algorithm. The original and modified training networks (modified with corresponding gene target expression data only) were used to determine ranks of target genes for each experiment. Raw data was obtained from published experiments (GEO) or conducted in in-house, as indicated in footnotes. Average ranks for duplicate experiments are shown. 


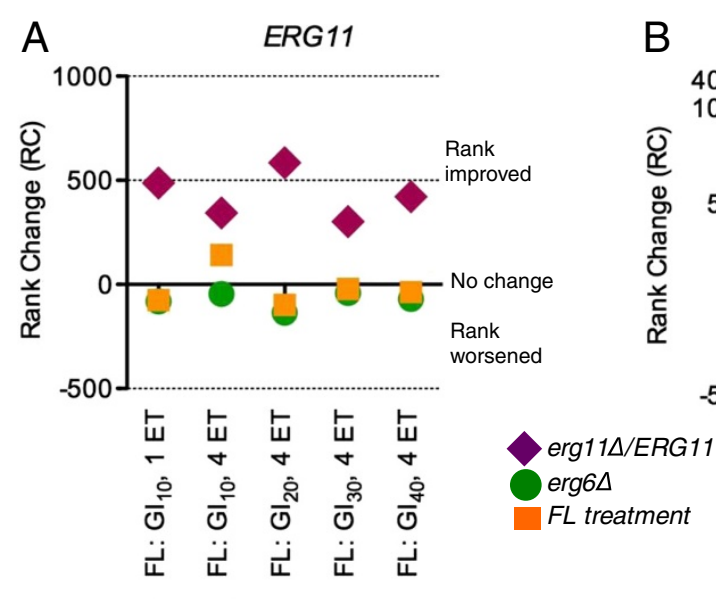

FL Treatment Experiment
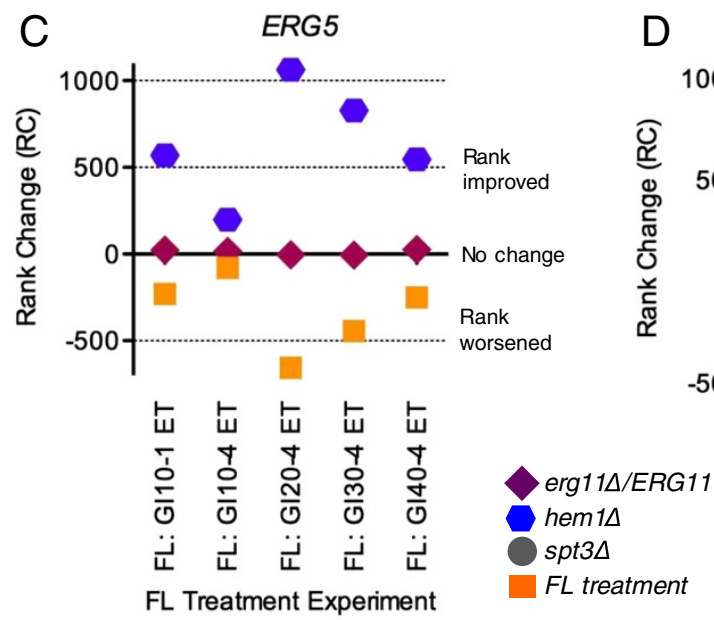

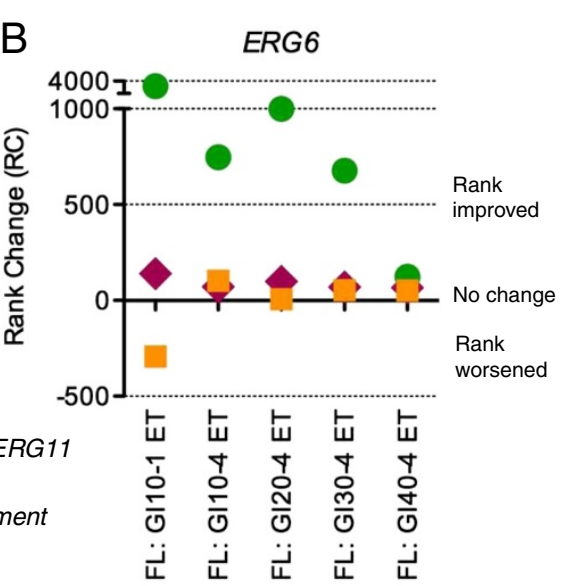

FL Treatment Experiment

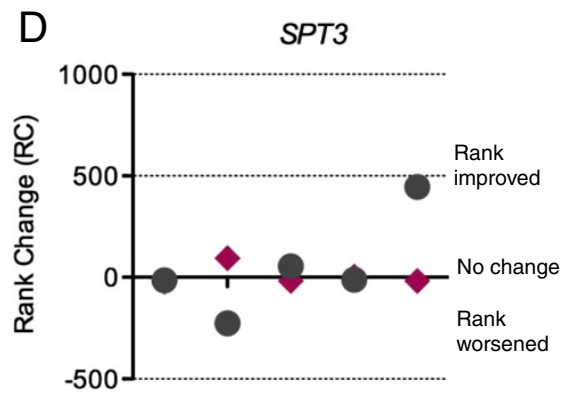

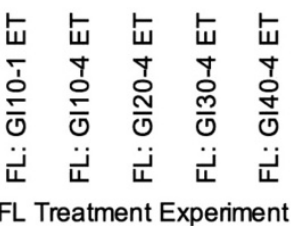

Figure 9 Training phase variation effects on single gene (rank) predictions. The modified training compendiums were used to predict ranks of FL-target genes, ERG11, ERG6, ERG5, and non-target gene, SPT3, in five representative FL treatment experiments. First, gene ranks for 2 replicate experiments were averaged. Next, ranks from the original training compendium were subtracted from ranks derived from the modified training compendium, yielding rank changes, or RCs. Finally, RCs (y-axis) were plotted for five representative FL treatment experiments ( $x$-axis) for each gene: (A) ERG11, (B) ERG6, (C) ERG5, and (D) SPT3. Positive RCs signified the gene rank improved with the addition of the corresponding deletion experiment data to the compendium. An RC of 0 indicated no change. A negative RC indicated rank increased or worsened.

expression data did not significantly alter SPT3 rank across the representative FL experiments; three of the ranks increased while two widely fluctuated (Figure 9D, Table 7).

Training phase: A further application of network

We explored if these findings were applicable to nocodazole (NOC), an antimitotic benzimidazole drug that destabilizes microtubules and causes cell cycle arrest in mitosis [48]. TUB1 is one of two functional genes (the other is TUB3) that encodes for $\alpha$-tubulin, an essential protein of the $\alpha, \beta$-tubulin heterodimer [49]. $\alpha, \beta$-tubulin polymerizes into microtubules, which are critical components of the mitotic and meiotic spindles and essential for cell division [49]. We hypothesized that addition of
Table 4 Comparison of rank changes (RCs) for ERG11 in network training phase

\begin{tabular}{|c|c|c|c|c|}
\hline \multirow[b]{3}{*}{ Expt analyzed } & \multicolumn{4}{|c|}{ ERG11 } \\
\hline & \multicolumn{2}{|c|}{+ Aerg11/ERG 11} & \multirow{2}{*}{$\begin{array}{l}+ \text { Aerg6 } \\
\text { Rank } \\
\text { change (RC) }\end{array}$} & \multirow{2}{*}{$\begin{array}{l}+\mathrm{FL} \\
\text { Rank } \\
\text { change (RC) }\end{array}$} \\
\hline & $\begin{array}{l}\text { Rank } \\
\text { change (RC) }\end{array}$ & Percentile & & \\
\hline $\mathrm{FL}_{\mathrm{Gl10}}, 1 \mathrm{DT}$ & 488 & 97 & -82 & -75 \\
\hline $\mathrm{FL}_{\mathrm{Gl10}}, 4 \mathrm{DT}$ & 344 & 92 & -45 & 142 \\
\hline $\mathrm{FL}$ G120, 4DT & 585 & 96 & -136 & -98 \\
\hline $\mathrm{FL}$ G130, 4DT & 301 & 88 & -41 & -21 \\
\hline $\mathrm{FL}_{\mathrm{Gl} \mid 40}, 4 \mathrm{DT}$ & 421 & 94 & -70 & -37 \\
\hline
\end{tabular}

RCs were reported for ERG11 across $5 \mathrm{FL}$ treatment experiments, under 3 different training compendiums: + $\Delta$ erg11/ERG11, + $\Delta$ erg6, and + FL treatment. Percentile of RC was computed as the percentage of genes with a RC as high or higher than the target gene. RC percentiles $\geq 95$ indicated significant perturbation as identified by SSEM-Lasso. 
Table 5 Comparison of rank changes (RCs) for ERG6 in network training phase

\begin{tabular}{|c|c|c|c|c|c|}
\hline \multirow[b]{3}{*}{ Expt analyzed } & \multicolumn{5}{|c|}{ ERG6 } \\
\hline & \multicolumn{2}{|c|}{$+\Delta e r g 6$} & \multicolumn{2}{|c|}{$+\Delta \operatorname{erg} 11 / E R G 11$} & \multirow{2}{*}{$\begin{array}{l}+\mathrm{FL} \\
\text { Rank change (RC) }\end{array}$} \\
\hline & Rank change (RC) & Percentile & Rank change (RC) & $\overline{\text { Percentile }}$ & \\
\hline $\mathrm{FL}_{\mathrm{G} \mid 10}, 1 \mathrm{DT}$ & 3795 & 100 & 139 & 73 & -292 \\
\hline $\mathrm{FL}_{\mathrm{G} \mid 10}, 4 \mathrm{DT}$ & 748 & 98 & 71 & 43 & 102 \\
\hline $\mathrm{FL}_{\mathrm{Gl}} \mathrm{O}, 4 \mathrm{DT}$ & 999 & 99 & 99 & 57 & 6 \\
\hline $\mathrm{FL}$ GI30, 4DT & 677 & 97 & 70 & 47 & 54 \\
\hline $\mathrm{FL}_{\mathrm{G} \mid 40}, 4 \mathrm{DT}$ & 123 & 48 & 67 & 45 & 52 \\
\hline
\end{tabular}

RCs were reported for ERG6 across $5 \mathrm{FL}$ treatment experiments, under 3 different training compendiums: + 4 erg6, + $\Delta$ erg11/ERG11, and + FL treatment. Percentile of $\mathrm{RC}$ was computed as the percentage of genes with a RC as high or higher than the target gene. RC percentiles $\geq 95$ indicated significant perturbation as identified by SSEM-Lasso.

microarray expression data from a $t u b 1 \Delta / T U B 1$ deletion strain could potentially improve TUB1 rank in NOC treatment experiments.

All experiments were performed in BY4741 strains using the same media and conditions that were used in FL experiments. We determined NOC treatment concentrations using dose-response curves and growth inhibitory values (GIs) as we did for FL. Studies have shown that $S$. cerevisiae haploid cells treated with NOC (6 to $15 \mu \mathrm{g} / \mathrm{mL}$, or 20 to $50 \mu \mathrm{M}$ ) rapidly disassemble microtubules in the majority of the cell population, resulting in mitotic arrest with intact nuclei $[48,50]$. We chose a concentration slightly lower, $16 \mu \mathrm{M}$, which corresponded to $\mathrm{GI}_{10}$, and incubation intervals of 3 and 4 ETs.

Interestingly, SSEM-Lasso did not predict TUB1 perturbation as well as it did for ERG11 with the original training set. Still, upon addition of TUB1 deletion expression data, ranks dropped by over 300, and TUB1 RCs were in the top $98 \%$ and $97 \%$ of all RCs for $\mathrm{GI}_{10}$, $3 \mathrm{ET}$ and $\mathrm{GI}_{10}$, $4 \mathrm{ET}$ experiments, respectively (Table 8 ).

\section{Discussion}

Impact of testing phase variations on SSEM-Lasso predictions of gene sets

SSEM-Lasso was originally developed to identify a directly targeted gene of a genetic or drug perturbation [12]. We sought to expand the algorithm's utility by testing its ability to predict drug perturbations over several biological conditions and on a set of biologically-related genes, rather than on a single gene target. This was the first study that analyzed SSEM-Lasso output using ROC curves and corresponding AUC values for a group of predefined genes.

FL treatment of $S$. cerevisiae results in inhibition of lanosterol demethylation and subsequent depletion of ergosterol and accumulation of toxic methylated sterol precursors. This results in cell membrane damage and impaired fatty acid and lipid biosyntheses [35,37]. FL coordinates to Erg11p's heme iron impairing cytochromerelated processes, such as mitochondrial respiration (reviewed in Parks, et al. 1995 [36]). We created a gene set based on these biological processes, called FL-interacters. Experimental variations in ET and concentration manifested perturbations that followed a clear trend and were specific to the FL-interacters gene set. An ET of at least four cell doublings in the presence of drug proved optimal for accurate prediction of FL-interacters (Figure 5) Concentration changes did not exert as strong of an effect as $\mathrm{ET}$, and any concentration between $\mathrm{GI}_{10}$ and $\mathrm{GI}_{40}$ proved optimal to induce target-gene perturbations (Figure 7). Our results demonstrated that experimental "input" variables could be successfully optimized to identify a set of related genes targeted by a drug. Our methodology could therefore provide an experimental platform for future studies aimed at predicting drug targets at the multi-gene or pathway level.

In principle, given the training/testing paradigm common to the core of statistical and machine learning methods in general, the spirit of the analyses carried out in this study may be similarly used in exploring the sensitivity of other methods of drug-target prediction methods.

Table 6 Comparison of rank changes (RCs) for ERG5 in network training phase

\begin{tabular}{|c|c|c|c|c|}
\hline \multirow[b]{3}{*}{$\begin{array}{l}\text { Expt } \\
\text { analyzed }\end{array}$} & \multicolumn{4}{|c|}{ ERG5 } \\
\hline & \multicolumn{2}{|c|}{ + Ahem 1} & \multirow{2}{*}{$\begin{array}{l}\text { + Aerg11/ERG11 } \\
\text { Rank } \\
\text { change (RC) }\end{array}$} & \multirow{2}{*}{$\begin{array}{l}+\mathrm{FL} \\
\text { Rank } \\
\text { change (RC) }\end{array}$} \\
\hline & $\begin{array}{l}\text { Rank } \\
\text { change (RC) }\end{array}$ & Percentile & & \\
\hline $\mathrm{FL}_{\mathrm{Gl} 10}, 1 \mathrm{DT}$ & 569 & 97 & 23 & -230 \\
\hline $\mathrm{FL}_{\mathrm{Gl10}}, 4 \mathrm{DT}$ & 199 & 75 & 16 & -80 \\
\hline $\mathrm{FL}_{\mathrm{Gl} 20}, 4 \mathrm{DT}$ & 1064 & 97 & -5 & -656 \\
\hline $\mathrm{FL}_{\mathrm{GI} 30}, 4 \mathrm{DT}$ & 828 & 95 & -6 & -444 \\
\hline $\mathrm{FL}_{\mathrm{Gl} \mid 40}, 4 \mathrm{DT}$ & 547 & 90 & 26 & -251 \\
\hline
\end{tabular}

RCs were reported for ERG5 across $5 \mathrm{FL}$ treatment experiments, under 3 different training compendiums: + $\Delta$ hem $1,+\Delta$ erg11/ERG11, and + FL treatment. Percentile of RC was computed as the percentage of genes with a $\mathrm{RC}$ as high or higher than the target gene. RC percentiles $\geq 95$ indicated significant perturbation as identified by SSEM-Lasso. 
Table 7 Comparison of rank changes (RCs) for SPT3 in network training phase

\begin{tabular}{llll}
\hline & \multicolumn{3}{c}{ SPT3 } \\
\cline { 2 - 3 } & \multicolumn{2}{c}{+ Uspt3 } & + Aerg11/ERG11 \\
\cline { 2 - 3 } Expt analyzed & Rank change (RC) & Percentile & Rank change (RC) \\
\hline $\mathrm{FL}_{\mathrm{G} \mid 10}, 1 \mathrm{DT}$ & -15 & $\mathrm{n} / \mathrm{a}$ & -23 \\
$\mathrm{FL}_{\mathrm{G} \mid 10}, 4 \mathrm{DT}$ & -226 & $\mathrm{n} / \mathrm{a}$ & 95 \\
$\mathrm{FL}_{\mathrm{G} \mid 20}, 4 \mathrm{DT}$ & 55 & 63 & -17 \\
$\mathrm{FL}_{\mathrm{G} \mid 30}$ 4DT & -12 & $\mathrm{n} / \mathrm{a}$ & 4 \\
$\mathrm{FL}_{\mathrm{G} \mid 40}$ 4DT & 445 & 98 & -18 \\
\hline
\end{tabular}

RCs were reported for SPT3 across 5 FL treatment experiments, under 3 different training compendiums: $+\Delta$ spt 3 and $+\Delta$ erg11/ERG11. Percentile of $\mathrm{RC}$ was computed as the percentage of genes with a $\mathrm{RC}$ as high or higher than the target gene. RC percentiles $\geq 95$ indicated significant perturbation as identified by SSEM-Lasso.

However, researchers should be aware that optimal experimental conditions determined in this study are exquisitely linked to SSEM-Lasso and FL. Drugs with different modes-of-action, potencies, and potential for off-target effects may have dissimilar effects on gene expression under these reported experimental conditions. Further, FL effects could manifest dissimilar genetic perturbations when analyzed with a new statistical model. Finally, depending on the algorithm or supervised learning method, results may be more or less sensitive to experimental changes than those reported with SSEM-Lasso. Therefore, the approach would need to be adapted to the specifics of the measurements at hand, which have tended to vary in the literature (e.g., here we utilize only microarray expression profiles). Nevertheless, this study provides a good starting point for researchers to obtain accurate drug target predictions using microarray technology.

\section{Impact of testing phase variations on SSEM-Lasso drug target predictions at the single gene level}

In our study, SSEM-Lasso accurately predicted FL single gene targets upon drug treatment, yet experimental variables did not dramatically affect biological outcomes. With the exception of ERG6 (which showed a distinct rank improvement after two cell cycles of FL treatment), ERG11, UPC2, and HAP1 ranks did not significantly fluctuate over varying treatment conditions.

Table 8 Nocodazole study results in network training phase

\section{TUB1}

$+\triangle t u b 1 / T U B 1$

\begin{tabular}{llll}
\cline { 2 - 4 } Expt analyzed & Rank & RC & Percentile \\
\hline NocGl $_{10}, 3 \mathrm{DT}$ & 4283 & 333 & 98 \\
NocGl $_{10}, 4 \mathrm{DT}$ & 4640 & 371 & 97
\end{tabular}

Two nocodazole (NOC) treatment experiments were performed and RCs of a NOC target gene, TUB1, were evaluated. Ranks listed in table were the single values each obtained from one treatment experiment.
FL is known to target $E R G 11$ (primary), ERG6, UPC2, and $H A P 1$, so these genes were tracked to evaluate optimal experimental conditions. Unlike gene set predictions, SSEM-Lasso prediction of ERG11 perturbation was relatively unchanging over multiple testing phase modifications (Tables 1 and 2). These results demonstrated that changes in ERG11 expression caused by changes in ET and concentration were not significantly detected by SSEM-Lasso above the background genegene interaction "patterns" in the network. ERG6 expression, on the other hand, responded to longer FL exposure times, with four ETs yielding an optimal ERG6 rank in the top $87 \%$ of ranked genes (Table 1). Furthermore, ERG6 ranked consistently lower than ERG11 across all FL concentration experiments (Figure 8B, Table 2). $E R G 6$ encodes for $\Delta[24]$-sterol C-methyltransferase, an enzyme downstream of Erg11p that synthesizes fecosterol, an important precursor to ergosterol (Figure 2). FL effects may have manifested more prominently on ERG6 than on ERG11 because of the cell's reliance on Erg6p for pleiotropic cellular processes, including membrane rigidity and permeability, genetic transformation, conjugation and tryptophan uptake $[42,43]$. Furthermore, Erg6p places a high demand on the cell for metabolic energy, 12-14 ATP equivalents, to perform transmethylation reactions [36]. Another reason ERG6 may have ranked lower than ERG11 involves the unnatural accumulation of lanosterol and/or the depletion of ergosterol as a result of Erg11p inhibition by FL. Disruption of later stage ergosterol biosynthesis enzymes, like Erg6p, has been attributed to the build up of reactant metabolites along the ergosterol pathway [61]. Furthermore, genome-wide microarray studies have found the most responsive genes to ketoconaozle function downstream of ERG11, suggesting their induction is induced by ergosterol depletion [61].

Metabolic vs. transcription factor gene target predictions A different trend, in comparison to metabolic enzymes ERG11 and ERG6, was observed for transcription factors, UPC2 and HAP1. Upc2p induces the transcription of ergosterol biosynthesis genes upon sterol depletion [44,45]. Previous microarray studies in S. cerevisiae had identified induced UPC2 expression in response to ketoconaozle [62]. This result was consistent with the FLinduced UPC2 perturbation observed in this study. SSEM-Lasso pinpointed UPC2 dysregulation by $\mathrm{FL}$, with ranks in the $99^{\text {th }}$ percentile at four ETs and a range of concentrations (Tables 1 and 2). Depending on the presence of heme, Hap1p can bind the promoters of genes to activate or repress their expressions for aerobic or hypoxic growth, such as the CYP450 enzymes, ERG11 and ERG5 [48]. Hence, ERG gene expressions are highly dependent on HAP1 gene interactions and the aerobic 
state of the cell. Additionally, Hap1p represses transcription of its own gene by at least 20 -fold in a heat shock protein-dependent manner [63]. Ranks for HAP1 were comparable to UPC2 ranks, all performing significantly better than other genes at three and four ETs at higher FL concentrations (Tables 1 and 2). To factor perturbations better than metabolic enzymes in the context of a drug treatment.

We discovered that the type of molecular target metabolic enzyme or transcription factor, upstream or downstream - can play a large role in the overall performance of the algorithm.

\section{Training phase variables significantly improve single gene target predictions}

The goal of SSEM-Lasso's training phase was to infer a gene interaction network that filtered out gene-gene regulatory "patterns" so that genes experiencing a true additive shift in their transcript signals emerged above the gene network background. In this study, modified training compendiums, the training phase "variables", were created by adding of new gene deletion data to the training compendium. Each modified training compendium shifted the network's internal gene regulatory influences so that SSEM-Lasso more accurately predicted single gene targets. This was evidenced by significant rank decreases for ERG11, ERG6 and ERG5 upon addition of $\operatorname{erg} 11 \Delta / E R G 11$, erg6 , and hem1 data, respectively, to the training compendium (Figure 9A-C, Tables 4, 5 and 6). The erg6 $\Delta$ haploid deletion induced the most pronounced decrease in ERG6 rank predictions. This could be a result of more potent and specific transcriptional effects of the erg6 $\Delta$ knockout compared to the partial knockout, erg11 $/ E R G 11$, or the hem1 strain, which was shown to induce widespread transcriptional effects on many cellular processes [59].

Additional studies involving NOC treatment and TUB1 expression supported these FL findings. Rank changes for NOC target, TUB1, dropped significantly when the original training compendium was modified with tub1 $\triangle / T U B 1$ deletion data (Table 8). Only one replicate experiment of two NOC conditions, $\mathrm{GI}_{10} 3 \mathrm{DT}$ and $\mathrm{GI}_{10} 4 \mathrm{DT}$, was performed for this drug target study, which is a limitation to the analysis. Finally, rank improvements of a non-FL-targeted gene, such as SPT3, were not manifested with addition of corresponding haploid spt3 3 expression data to the compendium (Figure 9D). When taken together, these results indicated that gene targets and the modified training compendium should be specific to the drug under investigation in order to improve SSEM-Lasso's performance. Still, further training phase variations concerning other drug treatments and deletion strains, and including more replicates, are necessary to assess the applicability of this strategy to various drug-target predictions.

It was initially hypothesized that addition of FL treatment expression data to the training compendium could also minimize background gene-gene interactions specific to FL's effects. This was not the case, as inclusion of the $\mathrm{FL}$ experiment, $\mathrm{GI}_{40}$, $4 \mathrm{ET}$, modified gene regulatory "patterns" in a negative manner, increasing ranks for FL targets. This led to the conclusion that genetic knockouts produced more distinct and local effects on the network as opposed to "noiser" and potentially off-target perturbations induced with an exogenous compound. Additionally, S. cerevisiae is susceptible to "neighboring gene effects", whereby the phenotype of a particular strain (e.g., deletion strain) could be due to the effect the perturbation exerts not on the target gene, but on an "adjacent" gene [64]. With around $10 \%$ of all yeast genes experiencing this type of influence [64], it was possible that there were too many "neighboring" expression changes induced by a foreign compound, and thus "noise" was enhanced and direct FL gene targets were pushed further into the background.

The training phase variables caused dramatic improvements in SSEM-Lasso performance compared to the minor rank fluctuations observed for experimental testing variations. Thus, when examining a drug's potential effects using microarray data and our methodology, the experimental conditions played a more important role in predicting a gene set. On the other hand, fine-tuning of the computational variable, i.e. the gene interaction network, proved more critical for accurate predictions of single gene drug targets.

\section{SSEM-Lasso utility for the prediction of unknown drug targets}

These results suggest a unique, two-stage approach to predict an unknown drug target using genome-wide microarray data and a network-inference model, such as SSEM-Lasso. First, detection of one or more perturbed gene sets should hone in on one or more biological pathways affected by the drug in question. In the case of SSEM-Lasso, these pathways were set a priori by the experimentalist, permitting a more focused analysis of drug-induced effects. Once a target pathway is elucidated, genetic deletion data specific to genes along the target pathway can be added to the training data. Single gene results can then be analyzed under different network training conditions and compared to arrive at more accurate drug gene target predictions. Genes experiencing greater rank changes with the addition of their respective genetic deletion data to the training compendium can be considered more likely candidate drug targets. 


\section{Conclusions}

The goal of this study was to improve our understanding of the interaction between biological testing and computational training variables in order to produce more accurate predictions of drug action at the transcriptional level. Previously, the network-inference model, SSEMLasso, predicted molecular targets of genetic mutations more accurately than drug treatments. We discovered a two-stage approach that addressed this issue and outlined improved experimental and computational conditions for predictions of first, drug pathway and second, single gene targets. Experimentally, the duration cells are in contact with a compound and the changes in compound concentration do not have a dramatic effect on single gene targets. However, these variables must be optimized for more accurate target predictions at the gene set/pathway level. Computationally, addition of specific biologically-motivated expression data to the interaction network can influence the gene regulatory effects in a manner that better resolves perturbations at the single gene level. With these variables in mind, SSEM-Lasso, and by extension, similar computational methods, can be a tremendously useful tool for therapeutic discovery when implemented under the appropriately informed testing and training conditions.

\section{Methods}

\section{Yeast strains and treatment conditions}

All FL treatment experiments maintained constant background, growth conditions, and mRNA preparation and hybridization procedures. The wild-type $S$. cerevisiae

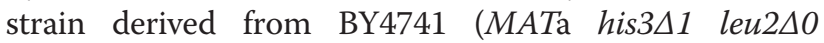
met $15 \Delta 0$ ura3 $\Delta 0$ ) was used for all FL treatments. This cell line has been used for systematic sequencing and deletion projects in which open reading frames were replaced by kanamycin cassettes (KanMX) to generate haploid and heterozygous knockouts [44]. Heterozygous knockout strains, ergll $\Delta / E R G 11$ and tubl $\triangle / T U B 1$ in a BY4743 background (MATa/MATa his $3 \Delta 1 /$ his $3 \Delta 1$ leu $2 \Delta 0 /$ leu $2 \Delta 0$ lys $2 \Delta 0 /+$ met $15 \Delta 0 /+$ ura $3 \Delta 0 /$ ura $3 \Delta 0$ ), and haploid strain, erg6 $6 \Delta$ in a BY4741 background were generated by the Saccharomyces genome deletion project [45] (Invitrogen) and used for genetic deletion microarray experiments. Haploid strain, hem1 $\Delta$ in a BY4742

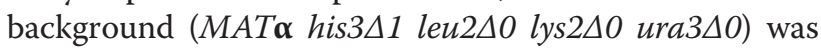
cultured under heme depletion conditions and microarray hybridization was performed according to Protchenko et al., 2008 [46].

Yeast growth is typically measured in cell population doubling times, determined by turbidity [65]. Consequently, a treatment collection time point, or exposure time (ET), was defined as the time it took a cell population to double in the presence of FL. ETs were longer than a typical 90-minute doubling time for wild-type $S$. cerevisiae cells at $30^{\circ} \mathrm{C}$, an anticipated result attributed to $\mathrm{FL}$ fungistatic activity.

\section{Determination of treatment concentrations}

To determine FL and NOC growth inhibitory value (GI), a single colony of wild-type cells was inoculated into $10 \mathrm{~mL}$ YPD media ( $1 \%$ yeast extract, $2 \%$ bacto-peptone, $2 \%$ dextrose) overnight, diluted with YPD medium to give an $\mathrm{OD}_{600}$ of 0.005 , and pipetted $(200 \mu \mathrm{l})$ into a flat-bottom 96-well plate. Serial dilutions of FL (Sigma) or NOC (Calbiochem) were prepared in 100\% DMSO and added $(5 \mu \mathrm{L})$ to cells to obtain final concentrations of $200 \mu \mathrm{M}$ $10 \mu \mathrm{M}$ (for FL) and $60-10 \mu \mathrm{M}$ (for NOC). Each concentration was represented $6 \mathrm{x}$ on a single plate. The plates were incubated at $30^{\circ} \mathrm{C}$ overnight and the $\mathrm{OD}_{600}$ of control and treated wells were determined. Control absorbance values were normalized to $0 \%$ inhibition. Doseresponse curves were generated by plotting the \% growth inhibition (final) versus the $\log _{10}$ (drug concentration). GI percentages $(0.5,5,10,20,30,40)$ were then determined with GraphPad using the four parameter fit model.

\section{Genome-wide microarrays}

A single colony of wild-type or deletion yeast strains was inoculated into $10 \mathrm{ml}$ YPD media overnight, then diluted with YPD medium to give an $\mathrm{OD}_{600}$ between 0.08 and 0.1 . G418 was used for KanMX selection conditions of the deletion strains (final concentration $200 \mathrm{mg} / \mathrm{L}$ ). Cells were immediately treated with the appropriate concentration of FL, NOC, or DMSO (final concentration $1 \mathrm{v} / \mathrm{v} \% \mathrm{DMSO}$ ), incubated at $30^{\circ} \mathrm{C}$ with shaking $(250 \mathrm{rpm})$, and collected at mid-log growth phase for one, two, three, or four ETs. Cells were harvested by centrifugation at $500 \mathrm{x} \mathrm{g}, 5$ minutes, room temperature, flash frozen and stored at $-80^{\circ} \mathrm{C}$. Total RNA was isolated using the acid phenol chloroform method. Briefly, cell pellets were thawed, re-suspended in lysis buffer, and RNA was extracted with hot acid phenol: chloroform (Fisher). After three extractions, the supernatant, containing RNA, was added to $100 \%$ cold ethanol, and RNA was allowed to precipitate at $-20^{\circ} \mathrm{C}$ for 4-6 hours. RNA was pelleted and washed with $70 \%$ ethanol before dissolving in DEPC-treated water. Poly $(\mathrm{A})^{+} \mathrm{RNA}$ was next isolated using Oligotex mRNA kit (Qiagen Inc.) and amplified and hybridized to Affymetrix YG S98, except only 25 ng of mRNA was used. Raw expression data was RMAnormalized and processed with SSEM-Lasso.

\section{Establishing gene sets}

Relationships between genes in a gene set were based on published literature and biological pathway and yeast genome databases, Kyoto Encyclopedia of Genes and Genomes (KEGG) and Gene Ontology (GO). Each gene set contained between 72 and 74 genes. See Additional files for specific genes. 


\section{SSEM-Lasso algorithm and predictions of gene targets}

Methods of Cosgrove et al., 2008 [12] were adopted to identify gene perturbations using Lasso regression in a sparse simultaneous equation model (SSEM-Lasso). We briefly sketch the key elements of this approach here; refer the reader to Cosgrove et al., 2008 [12] for full details. With SSEM-Lasso, the mean level of gene expression from a single gene is described as a function of two elements (1) the gene expression of all other genes in a network and (2) an external perturbation parameter. The notion of a "targeted gene" refers to an external perturbation to the mean mRNA level of a gene that cannot be explained by gene-gene interactions alone. For $\mathrm{p}$ genes and $\mathrm{n}$ observations, the model can be written as: $Y=B Y+\Phi+E$, where $Y$ is a p by matrix of gene transcript measurements (for $\mathrm{p}$ genes in $\mathrm{n}$ samples), $B$ is a $\mathrm{p}$ by p matrix of gene-gene interaction effects whose diagonal elements are fixed to zero (gene interaction network/matrix), $\Phi$ is a p by $\mathrm{n}$ matrix of external perturbations (derived from the experiment being tested) and $E$ is a p by $\mathrm{n}$ matrix of random noise assumed Gaussian with zero mean.

Note that the model used in SSEM-Lasso is an autoregressive model, with the variable $Y$ serving as both response and predictor. Auto-regressive models have a long history in traditional time series and spatial data analysis [66], and in recent years have proven popular for network-based modelling as well [67] (See Kolaczyak, 2009 Ch7.3). The manner in which we write the model above is a standard and concise representation [66]. In this form, the relationship among the gene expression is summarized (1) across all microarrays (i.e., it is a multivariate statistical representation, involving the entire matrix $Y$, rather than a single column), and (2) at the level of the joint marginal distribution within each microarray (i.e., it involves the full columns in $Y$, rather than just their individual elements). However, at the level of an individual measurement in $Y$, say single gene $\mathrm{k}$ in sample i, denoted as $y_{k i}$, the model may be shown to specify that the conditional distribution of $y_{k i}$, given all other genes $\mathrm{j} \neq \mathrm{k}$ in experiment $\mathrm{i}$, denoted as $y_{j i}$, is of the form

$$
y_{k i}=\sum_{j \neq K} B_{k j} y_{j i}+\phi_{k i}+e_{k i}
$$

That is, conditionally, the expression of any one gene is modelled as a linear combination of that of the others, plus a possible perturbation, plus a noise term [12]. Thus, this type of model is a natural way of capturing the notion of the expression levels of each gene being influenced by the expression levels all other genes. Additionally, this conditional form shows why a regression-based strategy is natural for estimating the unknown parameters in $B$.
As in Cosgrove et al., 2008, the method was implemented in two steps. First, the original training compendium of RMA-normalized Affymetrix data was used to infer the gene-gene interaction network $\mathrm{B}$. This training compendium consisted of 1039 Affymetrix YG S98 GeneChips, representing 465 experimental conditions [12]. A simpler, simultaneous equation model was assumed by setting $\Phi$ to zero and estimating B rowby-row using a sparse regression technique.

Lasso regression is a form of penalized regression, in which the standard least squares goodness-of-fit criterion is augmented with an additional term capturing the sum of the absolute values of all regression coefficients in the model. Such penalties, developed and studied extensively over the past two decades, are known to encourage sparse models and are particularly useful in contexts (such as the current one) in which a relatively small number of variables (i.e., genes) need to be selected from among a very large number. See Cosgrove et al., 2008 [12] for additional details on implementation of the Lasso methodology, and [68], for a formal characterization of the performance of SSEM-Lasso (including the expected accuracy of the Lasso-based regression), both theoretical and under simulation.

In the second step of the SSEM-Lasso method, using the estimate of B resulting from the first step, an outlier analysis of the residuals is conducted: $\hat{r}^{\text {pert }}=y^{\text {pert }}-B y^{\text {pert }}$, where $\mathrm{y}^{\text {pert }}$ is a $\mathrm{p} x 1$ vector of expression values across $\mathrm{p}$ genes in a single experiment (in our case a FL experiment). The residual, $\hat{r}^{\text {pert }}$ is a combination of the external influence $\Phi^{\text {pert }}$ of the perturbation and noise. Residuals were then ranked by their absolute values for all annotated yeast genes (1-6681). Genes with low ranks (and thus high residuals) were genes that SSEM-Lasso distinguished from the gene network background and were considered potential targets of the applied perturbation.

\section{Modification of the training compendium}

For FL training phase modifications, raw expression data from two, individual microarray experiments performed under identical conditions (e.g. two replicates of an Affymetrix GeneChip from an erg11D/ERG11 heterozygous mutant experiment) were RMA-normalized. Expression data were input into the training compendium as a single experiment file. A new, distinct training network was inferred as described above. In this way, the gene interaction matrix acted as the variable for the training phase modification experiments. RMA-normalization for all modified training compendiums was done in one step and included GeneChips from the original training compendium [12] plus additional experiments: erg11 / ERG11, erg64, hem1A (GSM241150 and GSM241150), spt3A (GSM239658 and GSM239659), Fluconazole 
treatment, rpl7aA (GSM217617 and GSM217618) and Pterostilbene treatment (GSM266726 and GSM266728).

\section{Determining gene ranks, rank changes, and percentiles} After implementing SSEM-Lasso, gene ranks for all FL treatment experiments were obtained and averaged for each experimental condition (see Background and Figure 1). Gene ranks for NOC experiments were single experiments from each condition. All ranks fell between 1 and 6681, which were the total number of genes in the compendium. The percentile of a target gene in the testing phase was computed by dividing the total number of genes with ranks less than the target gene by the total number of genes (6681). Rank changes (RCs) were computed for the training phase by subtracting the rank obtained with the modified training compendium from the rank obtained with the original compendium. The $\mathrm{RC}$ percentile of a target gene in the testing phase was similarly computed.

\section{Additional files}

Additional file 1: (Previous_Predictions_SSEMLasso.pdf) - Previous SSEM-Lasso predictions of genetic and drug gene target perturbations. SSEM-Lasso gene ranks are listed for gene targets of genetic deletions and bioactive compound/drug treatments. All microarray experiments were performed using Affymetrix Yeast Genome 98 gene chips, and data was RMA-normalized before processing with SSEM-Lasso. Experiments cited were published, annotated or conducted in in-house. Average ranks for experiments that provided replicates are shown.

Additional file 2: (FL-interacters_Gene_Set.pdf) - FL-interacters Gene Set.

Additional file 3: (DNA_Gene_Set.pdf) - Orthogonal Gene Set: DNA replication and repair.

Additional file 4: (Pyrimidine Gene Set.pdf) - Orthogonal Gene Set: Pyrimidine biosynthesis and metabolism.

Additional file 5: (RNA_Gene_Set.pdf) - Orthogonal Gene Set: RNA transport.

Additional file 6: (Glyc_Pent_Gene_Set.pdf) - Orthogonal Gene Set: Glycolysis and pentose phosphate.

Additional file 7: (Mitosis.pdf) - Orthogonal Gene Set: Mitosis.

\section{Competing interests}

The authors declare that they have no competing interests.

\section{Authors' contributions \\ LC conducted all biological assays and computational and qualitative analyses of SSEM-Lasso results. LC wrote the manuscript. LP processed micro- array expression data through SSEM-Lasso, generated ROC curves, and con- tributed to the statistical methods and explanations in the manuscript. EK and SES conceived this study as a progression of a previous SSEM-Lasso study, conducted by Elissa Cosgrove (EC). EC and Yingchun Zhou developed SSEM-Lasso for gene target identification studies. All authors read and ap- proved the final manuscript.}

\section{Acknowledgements}

This research was supported, in part, by National Institutes of Health Grant GM078987, National Science Foundation Integrative Graduate Education and Research Traineeship Fellowship DGE-0654108.

\section{Author details}

'Department of Chemistry, Boston University, Boston, MA, USA. ${ }^{2}$ Program in Bioinformatics and Mathematics, Boston University, Boston, MA, USA. ${ }^{3}$ Department of Mathematics and Statistics, Boston University, Boston, MA, USA

Received: 29 August 2012 Accepted: 21 November 2013 Published: 20 January 2014

\section{References}

1. Debouck C, Goodfellow PN: DNA microarrays in drug discovery and development. Nat Genet 1999, 21(1 Suppl):48-50.

2. Gerhold DL, Jensen RV, Gullans SR: Better therapeutics through microarrays. Nat Genet 2002, 32(Suppl):547-551.

3. Ho CH, Piotrowski J, Dixon SJ, Baryshnikova A, Costanzo M, Boone C: Combining functional genomics and chemical biology to identify targets of bioactive compounds. Curr Opin Chem Biol 2011, 15(1):66-78.

4. Meltzer PS: Spotting the target: microarrays for disease gene discovery. Curr Opin Genet Dev 2001, 11(3):258-263.

5. Oehler VG, Yeung KY, Choi YE, Bumgarner RE, Raftery AE, Radich JP: The derivation of diagnostic markers of chronic myeloid leukemia progression from microarray data. Blood 2009, 114(15):3292-3298.

6. Pe'er $D$, Hacohen N: Principles and strategies for developing network models in cancer. Cell 2011, 144(6):864-873.

7. Giaever G, Shoemaker DD, Jones TW, Liang H, Winzeler EA, Astromoff A Davis RW: Genomic profiling of drug sensitivities via induced haploinsufficiency. Nat Genet 1999, 21(3):278-283.

8. Hillenmeyer ME, Ericson E, Davis RW, Nislow C, Koller D, Giaever G: Systematic analysis of genome-wide fitness data in yeast reveals novel gene function and drug action. Genome Biol 2010, 11(3):R30.

9. Lum PY, Armour CD, Stepaniants SB, Cavet G, Wolf MK, Butler JS, Hinshaw JC, Garnier P, Prestwich GD, Leonardson A, Garrett-Engele P, Rush CM, Bard M, Schimmack G, Phillips JW, Roberts CJ, Shoemaker DD: Discovering modes of action for therapeutic compounds using a genome-wide screen of yeast heterozygotes. Cell 2004, 116(1):121-137.

10. Parsons AB, Brost RL, Ding H, Li Z, Zhang C, Sheikh B, Brown GW, Kane PM, Hughes TR, Boone C: Integration of chemical-genetic and genetic interaction data links bioactive compounds to cellular target pathways. Nat Biotechnol 2004, 22(1):62-69.

11. St Onge RP, Mani R, Oh J, Proctor M, Fung E, Davis RW, Nislow C, Roth FP, Giaever G: Systematic pathway analysis using high-resolution fitness profiling of combinatorial gene deletions. Nat Genet 2007, 39(2):199-206.

12. Cosgrove EJ, Zhou Y, Gardner TS, Kolaczyk ED: Predicting gene targets of perturbations via network-based filtering of mRNA expression compendia. Bioinformatics 2008, 24(21):2482-2490

13. Bansal M, Della Gatta G, Wierzbowski J, Gardner T, di Bernardo D: Discovering drug mode of action using reverse-engineered gene networks. Conf Proc IEEE Eng Med Biol Soc 2005, 5:4739-4742.

14. di Bernardo D, Thompson MJ, Gardner TS, Chobot SE, Eastwood EL, Wojtovich AP, Elliott SJ, Schaus SE, Collins JJ: Chemogenomic profiling on a genome-wide scale using reverse-engineered gene networks. Nat Biotechnol 2005, 23(3):377-383.

15. Gardner TS, di Bernardo D, Lorenz D, Collins JJ: Inferring genetic networks and identifying compound mode of action via expression profiling. Sci 2003, 301(5629):102-105.

16. Hallen K, Bjorkegren J, Tegner J: Detection of compound mode of action by computational integration of whole-genome measurements and genetic perturbations. BMC Bioinforma 2006, 7:51

17. Iorio F, Bosotti R, Scacheri E, Belcastro V, Mithbaokar P, Ferriero R, Murino L, Tagliaferri R, Brunetti-Pierri N, Isacchi A, di Bernardo D: Discovery of drug mode of action and drug repositioning from transcriptional responses. Proc Natl Acad Sci USA 2010, 107(33):14621-14626.

18. van Laarhoven T, Nabuurs SB, Marchiori E: Gaussian interaction profile kernels for predicting drug-target interaction. Bioinformatics 2011, 27(21):3036-3043.

19. Faulon JL, Misra M, Martin S, Sale K, Sapra R: Genome scale enzymemetabolite and drug-target interaction predictions using the signature molecular descriptor. Bioinformatics 2008, 24(2):225-233. 
20. Yamanishi Y, Araki M, Gutteridge A, Honda W, Kanehisa M: Prediction of drug-target interaction networks from the integration of chemical and genomic spaces. Bioinformatics 2008, 24(13):i232-240.

21. Jacob L, Vert JP: Protein-ligand interaction prediction: an improved chemogenomics approach. Bioinformatics 2008, 24(19):2149-2156.

22. Campillos M, Kuhn M, Gavin AC, Jensen $\amalg$, Bork P: Drug target identification using side-effect similarity. Sci 2008, 321(5886):263-266.

23. Chua HN, Roth FP: Discovering the targets of drugs via computational systems biology. J Biol Chem 2011, 286(27):23653-23658.

24. Tatonetti N, Liu T, Altman R: Predicting drug side-effects by chemical systems biology. Genome Biol 2009, 10(9):238.

25. Kim K, Zakharkin SO, Allison DB: Expectations, validity, and reality in gene expression profiling. J Clin Epidemiol 2010, 63(9):950-959.

26. Cosgrove E, Gardner T, Kolaczyk E: On the Choice and Number of Microarrays for Transcriptional Regulatory Network Inference. BMC Bioinforma 2010, 11(1):454.

27. Huttenhower C, Troyanskaya OG: Assessing the functional structure of genomic data. Bioinformatics 2008, 24(13):i330-338.

28. Kanehisa M, Goto S: KEGG: kyoto encyclopedia of genes and genomes Nucleic Acids Res 2000, 28(1):27-30.

29. Schomburg I, Chang A, Placzek S, Sohngen C, Rother M, Lang M, Munaretto C, Ulas S, Stelzer M, Grote A, Scheer M, Schomburg D: BRENDA in 2013: integrated reactions, kinetic data, enzyme function data, improved disease classification: new options and contents in BRENDA. Nucleic Acids Res 2013, 41(Database issue):D764-772.

30. Prabhakara S, Acharya R: SIMCOMP: a hybrid soft clustering of metagenome reads. In Proceedings of the 5th IAPR international conference on Pattern recognition in bioinformatics, Volume 1887866. Nijmegen, The Netherlands: Springer-Verlag; 2010:113-124.

31. Hitchcock CA, Dickinson K, Brown SB, Evans EG, Adams DJ: Interaction of azole antifungal antibiotics with cytochrome P-450-dependent 14 alphasterol demethylase purified from Candida albicans. Biochem J 1990, 266 (2):475-480

32. Yoshida $Y$, Aoyama $Y$ : Interaction of azole antifungal agents with cytochrome P-45014DM purified from Saccharomyces cerevisiae microsomes. Biochem Pharmacol 1987, 36(2):229-235.

33. Ghannoum MA, Rice LB: Antifungal agents: mode of action, mechanisms of resistance, and correlation of these mechanisms with bacterial resistance. Clin Microbiol Rev 1999, 12(4):501-517.

34. Kelly SL, Lamb DC, Corran AJ, Baldwin BC, Kelly DE: Mode of action and resistance to azole antifungals associated with the formation of 14 alpha-methylergosta-8,24(28)-dien-3 beta,6 alpha-diol. Biochem Biophys Res Commun 1995, 207(3):910-915.

35. Abe F, Usui K, Hiraki T: Fluconazole modulates membrane rigidity, heterogeneity, and water penetration into the plasma membrane in Saccharomyces cerevisiae. Biochem 2009, 48(36):8494-8504.

36. Parks LW, Casey WM: Physiological implications of sterol biosynthesis in yeast. Annu Rev Microbiol 1995, 49:95-116

37. Veen $M$, Lang $C$ : Interactions of the ergosterol biosynthetic pathway with other lipid pathways. Biochem Soc Trans 2005, 33(Pt 5):1178-1181.

38. Bard M, Lees ND, Turi T, Craft D, Cofrin L, Barbuch R, Koegel C, Loper JC Sterol synthesis and viability of erg11 (cytochrome P450 lanosterol demethylase) mutations in Saccharomyces cerevisiae and Candida albicans. Lipids 1993, 28(11):963-967.

39. Kalb VF, Woods CW, Turi TG, Dey CR, Sutter TR, Loper JC: Primary structure of the P450 lanosterol demethylase gene from Saccharomyces cerevisiae. DNA 1987, 6(6):529-537.

40. Turi TG, Loper JC: Multiple regulatory elements control expression of the gene encoding the Saccharomyces cerevisiae cytochrome P450, lanosterol 14 alpha-demethylase (ERG11). J Biol Chem 1992, 267(3):2046-2056.

41. Emter R, Heese-Peck A, Kralli A: ERG6 and PDR5 regulate small lipophilic drug accumulation in yeast cells via distinct mechanisms. FEBS Lett 2002, 521(1, Ӓi3):57-61.

42. Gaber RF, Copple DM, Kennedy BK, Vidal M, Bard M: The yeast gene ERG6 is required for normal membrane function but is not essential for biosynthesis of the cell-cycle-sparking sterol. Mol Cell Biol 1989, 9(8):3447-3456.

43. McCammon MT, Hartmann MA, Bottema CD, Parks LW: Sterol methylation in Saccharomyces cerevisiae. J Bacteriol 1984, 157(2):475-483.

44. Davies BS, Wang HS, Rine J: Dual activators of the sterol biosynthetic pathway of Saccharomyces cerevisiae: similar activation/regulatory domains but different response mechanisms. Mol Cell Biol 2005, 25(16):7375-7385.
45. Vik A, Rine J: Upc2p and Ecm22p, dual regulators of sterol biosynthesis in Saccharomyces cerevisiae. Mol Cell Biol 2001, 21(19):6395-6405.

46. MacPherson S, Akache B, Weber S, De Deken X, Raymond M, Turcotte B: Candida albicans zinc cluster protein Upc2p confers resistance to antifungal drugs and is an activator of ergosterol biosynthetic genes. Antimicrob Agents Chemother 2005, 49(5):1745-1752.

47. Oliver BG, Song $J$, Choiniere $J H$, White TC: cis-Acting elements within the Candida albicans ERG11 promoter mediate the azole response through transcription factor Upc2p. Eukaryot Cell 2007, 6(12):2231-2239.

48. Hickman MJ, Winston F: Heme levels switch the function of Hap1 of Saccharomyces cerevisiae between transcriptional activator and transcriptional repressor. Mol Cell Biol 2007, 27(21):7414-7424.

49. Zhang $L$, Hach A: Molecular mechanism of heme signaling in yeast: the transcriptional activator Hap1 serves as the key mediator. Cell Mol Life Sci 1999, 56(5-6):415-426.

50. Verdiere J, Gaisne M, Labbe-Bois R: CYP1 (HAP1) is a determinant effector of alternative expression of heme-dependent transcribed genes in yeast [corrected]. Mol Gen Genet 1991, 228(1-2):300-306.

51. Davies BSJ, Rine J: A Role for Sterol Levels in Oxygen Sensing in Saccharomyces cerevisiae. Genetics 2006, 174(1):191-201.

52. Kelly SL, Lamb DC, Baldwin BC, Corran AJ, Kelly DE: Characterization of Saccharomyces cerevisiae CYP61, sterol delta22-desaturase, and inhibition by azole antifungal agents. J Biol Chem 1997, 272(15):9986-9988.

53. Mueller $M$, de la Pena $A$, Derendorf $H$ : Issues in pharmacokinetics and pharmacodynamics of anti-infective agents: kill curves versus MIC. Antimicrob Agents Chemother 2004, 48(2):369-377.

54. Venisse N, Gregoire N, Marliat M, Couet W: Mechanism-based pharmacokinetic-pharmacodynamic models of in vitro fungistatic and fungicidal effects against Candida albicans. Antimicrob Agents Chemother 2008, 52(3):937-943.

55. Rodriguez RJ, Low C, Bottema CDK, Parks LW: Multiple functions for sterols in Saccharomyces cerevisiae. Biochim Biophys Acta (BBA) - Lipids and Lipid Metabolism 1985, 837(3):336-343.

56. Rosenfeld E, Beauvoit B: Role of the non-respiratory pathways in the utilization of molecular oxygen by Saccharomyces cerevisiae. Yeast 2003, 20(13):1115-1144.

57. Giaever G, Chu AM, Ni L, Connelly C, Riles L, Veronneau S, Dow S, Lucau-Danila A, Anderson K, Andre B, Arkin AP, Astromoff A, ElBakkoury M, Bangham R, Benito R, Brachat S, Campanaro S, Curtiss M, Davis K, Deutschbauer A, Entian KD, Flaherty P, Foury F, Garfinkel DJ, Gerstein M, Gotte D, Guldener U, Hegemann JH, Hempel S, Herman Z, et al: Functional profiling of the Saccharomyces cerevisiae genome. Nature 2002, 418(6896):387-391.

58. Winzeler EA, Shoemaker DD, Astromoff A, Liang H, Anderson K, Andre B, Bangham R, Benito R, Boeke JD, Bussey H, Chu AM, Connelly C, Davis K, Dietrich F, Dow SW, El Bakkoury M, Foury F, Friend SH, Gentalen E, Giaever G, Hegemann JH, Jones T, Laub M, Liao H, Liebundguth N, Lockhart DJ, Lucau-Danila A, Lussier M, M'Rabet N, Menard P, et al: Functional characterization of the $\mathrm{S}$. cerevisiae genome by gene deletion and parallel analysis. Sci 1999, 285(5429):901-906.

59. Protchenko O, Shakoury-Elizeh M, Keane P, Storey J, Androphy R, Philpott CC: Role of PUG1 in Inducible Porphyrin and Heme Transport in Saccharomyces cerevisiae. Eukaryot Cell 2008, 7(5):859-871.

60. James N, Landrieux E, Collart MA: A SAGA-Independent Function of SPT3 Mediates Transcriptional Deregulation in a Mutant of the Ccr4-Not Complex in Saccharomyces cerevisiae. Genetics 2007, 177(1):123-135.

61. Dimster-Denk D, Rine J, Phillips J, Scherer S, Cundiff P, DeBord K, Gilliland D, Hickman S, Jarvis A, Tong L, Ashby M: Comprehensive evaluation of isoprenoid biosynthesis regulation in Saccharomyces cerevisiae utilizing the Genome Reporter Matrix. J Lipid Res 1999, 40(5):850-860.

62. Agarwal AK, Rogers PD, Baerson SR, Jacob MR, Barker KS, Cleary JD, Walker LA, Nagle DG, Clark AM: Genome-wide expression profiling of the response to polyene, pyrimidine, azole, and echinocandin antifungal agents in Saccharomyces cerevisiae. J Biol Chem 2003, 278(37):34998-35015.

63. Hon T, Hach A, Tamalis D, Zhu Y, Zhang L: The yeast heme-responsive transcriptional activator Hap1 is a preexisting dimer in the absence of heme. J Biol Chem 1999, 274(32):22770-22774.

64. Ben-Shitrit T, Yosef N, Shemesh K, Sharan R, Ruppin E, Kupiec M: Systematic identification of gene annotation errors in the widely used yeast mutation collections. Nat Methods 2012, 9(4):373-378. 
65. Pringle JR, Mor JR: Methods for monitoring the growth of yeast cultures and for dealing with the clumping problem. Methods Cell Biol 1975, 11:131-168

66. Cressie N, Wikle CK: Statistics for Spatio-Temporal Data. Hoboken, NJ: Wiley; 2011.

67. Kolaczyk ED: Statistical Analysis of Network Data: Methods and Models. New York: Springer-Verlag New York; 2009.

68. Yang S, Kolaczyk ED: Target detection via network filtering. IEEE Trans Inf Theor 2010, 56(5):2502-2515.

doi:10.1186/1752-0509-8-7

Cite this article as: Christadore et al.: Improvement of experimental

testing and network training conditions with genome-wide microarrays for more accurate predictions of drug gene targets. BMC Systems Biology 2014877

\section{Submit your next manuscript to BioMed Central and take full advantage of:}

- Convenient online submission

- Thorough peer review

- No space constraints or color figure charges

- Immediate publication on acceptance

- Inclusion in PubMed, CAS, Scopus and Google Scholar

- Research which is freely available for redistribution 